\title{
Auditory-Dependent Vocal Recovery in Adult Male Zebra Finches Is Facilitated by Lesion of a Forebrain Pathway That Includes the Basal Ganglia
}

\author{
John A. Thompson, ${ }^{1,4}$ Wei Wu, ${ }^{2}$ Richard Bertram, ${ }^{1,3}$ and Frank Johnson ${ }^{1,4}$ \\ ${ }^{1}$ Program in Neuroscience and Departments of ${ }^{2}$ Statistics, ${ }^{3}$ Mathematics, and ${ }^{4}$ Psychology, Florida State University, Tallahassee, Florida $32306-1270$
}

\begin{abstract}
The integration of two neural pathways generates learned song in zebra finches. The vocal motor pathway (VMP) is a direct connection between HVC (proper name) and the robust nucleus of the arcopallium (RA), whereas the anterior forebrain pathway (AFP) comprises an indirect circuit from HVC to RA that traverses the basal ganglia. Partial ablation (microlesion) of HVC in adult birds alters the integration of VMP and AFP synaptic input within RA and destabilizes singing. However, the vocal pattern shows surprising resilience because birds subsequently recover their song in $\sim 1$ week. Here, we show that deafening prevents vocal recovery after HVC microlesions, indicating that birds require auditory feedback to restore/relearn their vocal patterns. We then tested the role of the AFP (basal ganglia circuit) in this feedback-based recovery by ablating the output nucleus of the AFP [lateral magnocellular nucleus of the anterior nidopallium (LMAN)]. We found that LMAN ablation after HVC microlesions induced a sudden recovery of the vocal pattern. Thus, the AFP cannot be the neural locus of an instructive/learning mechanism that uses auditory feedback to guide vocal recovery, at least in this form of adult vocal plasticity. Instead, the AFP appears to be the source of the variable motor patterns responsible for vocal destabilization. In part, auditory feedback may restore song by strengthening the VMP component of synaptic input to RA relative to the AFP component.
\end{abstract}

Key words: songbird; ablation; auditory feedback; vocal plasticity; sensorimotor; deafening

\section{Introduction}

The learned songs of passerine birds arise from the integration of two forebrain neural pathways, the vocal motor pathway (VMP) and the anterior forebrain pathway (AFP). Both pathways originate within HVC and both terminate in the premotor nucleus robust nucleus of the arcopallium (RA). The VMP consists of a population of HVC neurons that form synapses within RA (Fig. $1 A)$. A second, distinct population of HVC neurons project to area $\mathrm{X}$ (within avian basal ganglia), forming the first synaptic connection within the AFP. From area X, the pathway traverses the thalamus and then lateral magnocellular nucleus of the anterior nidopallium (LMAN) before terminating in RA (Fig. 1A). Excitatory input from both HVC (VMP) and LMAN (AFP) converge onto individual RA neurons (Mooney and Konishi, 1991), although in adult birds HVC synapses in RA far outnumber those from LMAN ( 20:1) (Canady et al., 1988).

Electrophysiological and behavioral evidence suggests that the VMP and AFP have distinct roles in vocal behavior. For example, neural activity within the VMP drives stereotyped adult song (Yu and Margoliash, 1996; Hahnloser et al., 2002), and complete ab-

Received June 22, 2007; revised Sept. 12, 2007; accepted Sept. 24, 2007.

This work was supported by National Institutes of Health Grants DC008028 (J.A.T.) and DC02035 (F.J.). We thank Ross Henderson and Paul Hendrick for the design and fabrication of the environmental chambers used in this study. Dr. Richard Hyson, Tiffanie Holloway, and Erika Tschinkel provided technical assistance and advice on this manuscript. We thank Dr. Ofer Tchernichovski for use of the SA + software.

Correspondence should be addressed to Frank Johnson, Program in Neuroscience, Florida State University, Tallahassee, FL 32306-1270. E-mail: johnson@psy.fsu.edu.

DOI:10.1523/JNEUROSCI.2853-07.2007

Copyright $\odot 2007$ Society for Neuroscience $\quad$ 0270-6474/07/2712308-13\$15.00/0 lation of either HVC or RA abolishes the production of learned vocalizations (Simpson and Vicario, 1990). In contrast, the AFP is implicated in vocal plasticity, both during vocal learning (Bottjer et al., 1984), and adult vocal change caused by altered sensory or motor function (Williams and Mehta, 1999; Brainard and Doupe, 2000).

Although AFP signaling is necessary for vocal plasticity, the underlying mechanisms of vocal change are unclear. Models of AFP function have been proposed and focused on different aspects of vocal plasticity. A "purposive variation model” suggests that the AFP directly influences vocal production, with LMAN driving the vocal-motor variability necessary for trial-and-error song learning by juvenile birds (Kao et al., 2005; Ölveczky et al., 2005). An "instructive model" suggests that the AFP uses auditory feedback to compare vocal output against a memorized auditory template, selecting and reinforcing the production of template-matched vocalizations by the VMP (Troyer and Doupe, 2000a,b; Troyer and Bottjer, 2001).

Previously, we tested the functional roles of VMP and AFP in adult zebra finches by reducing VMP synapses in RA [via bilateral partial ablation (microlesion) of HVC] without altering the number of AFP synapses in RA (Thompson and Johnson, 2007). We observed an initial increase in vocal variability, suggesting that the AFP continues to generate vocal-motor variation in adulthood (cf. Kao et al., 2005). However, all birds subsequently recovered their preoperative song, raising the possibility of auditory-dependent relearning of the vocal pattern.

Here, we tested whether recovery from HVC microlesions 


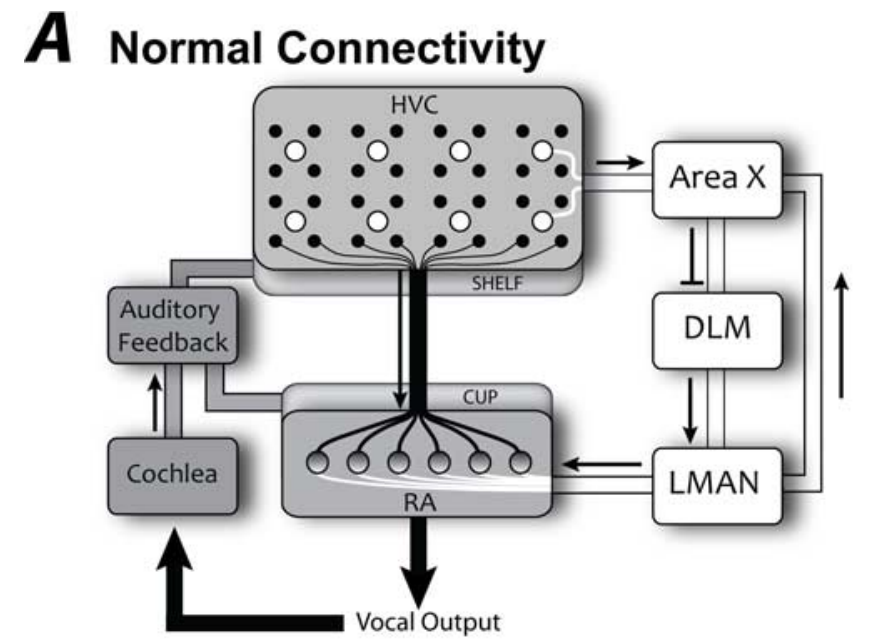

\section{B $\mathrm{HVCml}+$ Deaf}

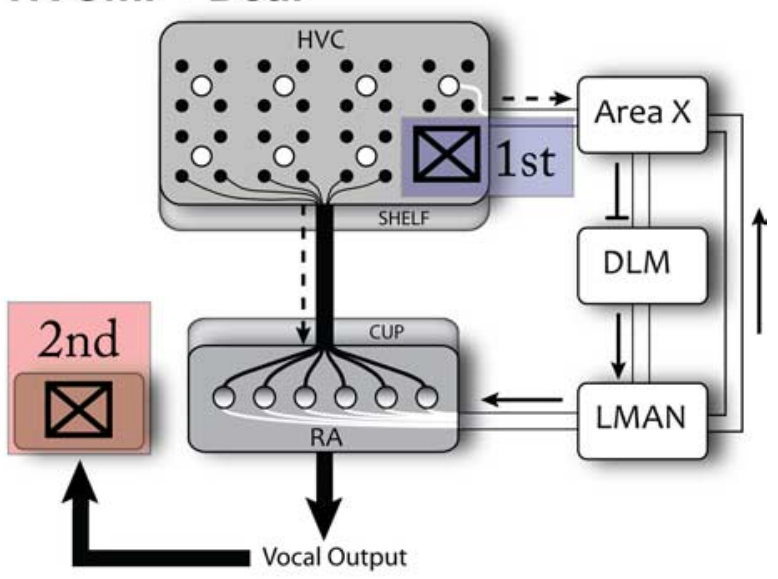

\section{C $\mathrm{HVCml}^{+}$LMAN Ablation}

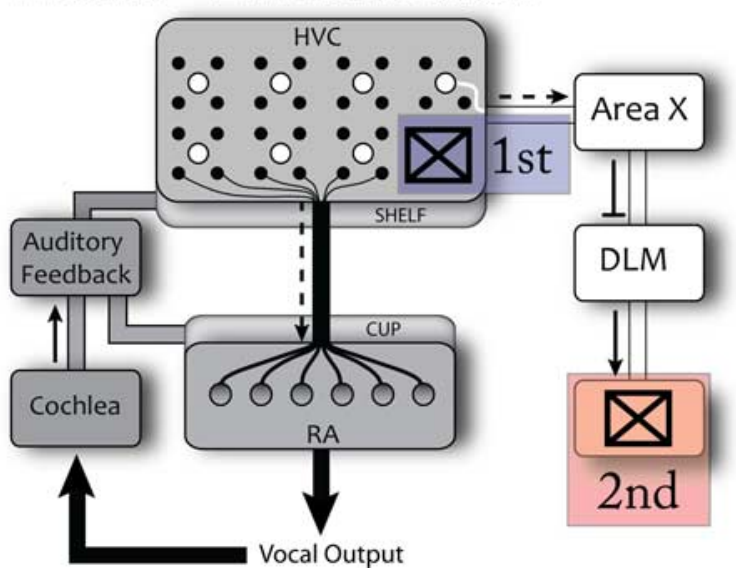

Figure 1. Simplified schematics of the song control circuit illustrate the hypotheses to be tested with emphasis on HVC and the integrated connectivity within RA. $A$, Projections from HVC to RA form the VMP, a pathway essential for production of the stereotyped adult vocal pattern (black pathway). A second pathway, the AFP (white pathway) originates in HVC and traverses basal ganglia (area X), thalamus [medial dorsolateral nucleus of the thalamus (DLM)], and the forebrain nucleus, LMAN, before terminating in RA. This circuit is important for juvenile vocal learning and adult vocal variability. Although the gray pathways (auditory feedback) terminate in the vicinity of both HVC and RA, HVC is the primary recipient of auditory information in the song control system (Vates et al., 1996; Coleman and Mooney, 2004). Within HVC, requires auditory feedback (Fig. $1 B$ ) and found that deafened adult birds fail to recover their vocal patterns. We then tested predictions from the two models for AFP function (Fig. 1C). LMAN ablation after HVC microlesions will either prevent recovery (instructional) or facilitate recovery (purposive variation). We found that LMAN ablation after HVC microlesions induced a sudden recovery of the vocal pattern, effectively ruling out the AFP as the neural locus of an instructive mechanism that guides adult vocal recovery.

\section{Materials and Methods}

\section{Animals and environment}

Twenty-eight adult male zebra finches ( $>120 \mathrm{~d}$ posthatch), either raised in our breeding colony or obtained from a commercial breeder, were individually housed in medium-sized bird cages $(26.67 \times 19.05 \times 39.37$ $\mathrm{cm})$ placed within computer-controlled environmental chambers $(75.69 \times 40.39 \times 47.24 \mathrm{~cm})$ that were fabricated by Florida State University Neuroscience Program engineering personnel. The design of the environmental chambers prevented visual as well as auditory access to other birds. A computer maintained both the photoperiod (14/10 h light/ dark cycle) and ambient temperature (set to $26^{\circ} \mathrm{C}$ ) within each chamber. Throughout the experiment, birds were provisioned daily with primarily millet-based assorted seed and tap water. Birds acclimated to the environmental chambers for 2 weeks before preoperative recordings and surgical manipulation. All daily care procedures and experimental manipulations of the birds were reviewed and approved by the Florida State University Animal Care and Use Committee.

\section{Surgeries}

All birds received two of the following surgeries: bilateral HVC microlesions (HVCml), bilateral cochlea removal (Deaf), bilateral LMAN ablation (LMAN Ablation), or bilateral control surgery (cs). For all birds, the first surgery followed a 2 week acclimation period and 3 preoperative recording days. The second surgery was performed after the first day of singing after the first surgery. After the second surgery, all birds survived for 3 weeks. The groups were as follows: $\mathrm{HVCml}+\operatorname{Deaf}(n=5)$, HVCcs + Deaf $(n=6), \mathrm{HVCml}+$ LMAN Ablation $(n=6), \mathrm{HVCml}+$ LMANcs $(n=6)$, and HVCcs + LMAN Ablation $(n=5)$. The procedures used for HVC microlesions and LMAN ablation were similar to those described by Thompson and Johnson (2007).

For both HVC microlesion and LMAN ablation surgeries, the following steps were taken: birds were deeply anesthetized with Equithesin $(0.04 \mathrm{cc})$, and then secured in a stereotaxic instrument. The skull was exposed by centrally incising the scalp and retracting the folds with curved forceps. After application of avian saline $(0.75 \mathrm{~g}$ of NaCl per 100 $\mathrm{ml}$ of $\mathrm{dH}_{2} \mathrm{O}$ ) to the exposed area, small craniotomies were placed over the approximate location of HVC or LMAN bilaterally. To determine the locations of these nuclei, the bifurcation at the midsagittal sinus was used as stereotaxic zero. After the surgery, the incision was treated with an antiseptic and sealed with veterinary adhesive, and the bird was returned to its home cage.

HVCml

Bilateral microlesions in HVC were placed by positioning an electrode (Teflon-insulated tungsten; $200 \mu \mathrm{m}$ diameter; A-M Systems, Everett,

RA-projecting neurons outnumber area $X$-projecting neurons by $>4: 1$ and both populations are distributed throughout the volume encompassed by HVC (Wild et al., 2005). Within RA, individual neurons receive synaptic input from both HVC and LMAN (Mooney and Konishi, 1991; Stark and Perkel, 1999); these inputs are nontopographic and topographic, respectively (Johnson et al., 1995; Foster and Bottjer, 1998). However, in the adult bird, VMP synapses within RA outnumber those of the AFP $(\sim 20: 1)$ (Canady et al., 1988). B, To test the role of auditory feedback during vocal recovery after HVC microlesions, birds were deafened by bilateral cochlea removal after HVC microlesions. C, HVC microlesions alter the integration in RA to favor AFP input, which may underlie the increase in vocal variability (Thompson and Johnson, 2007). To determine the role of the AFP in vocal recovery after HVC microlesions, birds received complete bilateral ablation of LMAN (the output nucleus of the AFP) after HVC microlesions. 
WA) directly lateral from stereotaxic zero with two penetrations per side. Predetermined coordinates for the location of HVC were used to estimate placement of lesion sites. The most medial lesion was $0.4 \mathrm{~mm}$ from the lateral penetration site, which was $2.4 \mathrm{~mm}$ from the midline. Each penetration had a depth of $0.6 \mathrm{~mm}$ with current set at $100 \mu \mathrm{A}$ for $35 \mathrm{~s}$.

The above coordinates produced microlesions that ablated $5-10 \%$ of the volume of each HVC, primarily within the lateral half of the nucleus. The potential effects of microlesion damage within HVC are informed by the following anatomical and behavioral considerations: Adult male zebra finch HVC contains interneurons and distinct populations of RAand X-projecting neurons; RA-projecting neurons outnumber area $\mathrm{X}$-projecting neurons by $>4: 1$, and both projection populations are distributed throughout the volume encompassed by HVC (Wild et al., 2005). Interneurons form local microcircuits connecting RA- and $\mathrm{X}$-projecting populations (Mooney and Prather, 2005). The axons of RAand X-projecting neurons appear to exit HVC caudoventrally and rostroventrally, respectively (Dutar et al., 1998; Mooney, 2000), and neither projection appears to be topographically organized (Foster and Bottjer, 1998). Afferent input to HVC also appears to lack topographic organization and derives primarily from ascending thalamic and telencephalic regions (Foster and Bottjer, 1998). Therefore, a microlesion placed within HVC will ablate a local population of interneurons and RA- and $\mathrm{X}$-projecting neurons, will disrupt local synaptic connectivity, and will disrupt the axonal connectivity of efferent and afferent pathways that may traverse the volume of the microlesion.

However, despite the anatomical disruption produced by HVC microlesions, behavioral evidence indicates that this damage does not interfere with the ability of HVC to generate a normal song pattern. HVC microlesions destabilize song only if LMAN is intact; with previous ablation of LMAN, HVC microlesions no longer destabilize the vocal pattern (Thompson and Johnson, 2007). Because ablation of LMAN alone produces a small improvement in song stereotypy (Kao et al., 2005), we infer that HVC microlesions lead to destabilized singing by weakening HVC input to RA, thereby favoring a vocal-variability signal carried to RA by LMAN. The data of Scharff et al. (2000) support this interpretation; using a laser ablation technique, these investigators observed vocal disruption only when RA-projecting neurons were selectively ablated. Selective ablation of X-projecting neurons had no effect on the vocal pattern.

\section{LMAN ablation}

Complete bilateral lesion of LMAN was accomplished with predetermined coordinates for optimal lesion placement. LMAN surgery involved eight penetrations, all of which had a depth of $2.6 \mathrm{~mm}$ : the first four were placed $3.8 \mathrm{~mm}$ anterior from zero, mirrored laterally at 1.4 and $1.9 \mathrm{~mm}$ from the midline. Two of the second four penetrations were placed more anterior $(5.4 \mathrm{~mm})$ from zero and positioned at $1.6 \mathrm{~mm}$ from the midline (which is midway between the first two lateral positions). The other two penetrations were more posterior $(2.2 \mathrm{~mm})$ from zero and again $1.6 \mathrm{~mm}$ lateral from the midline. The first four lesions were $3.5 \mathrm{~min}$ in duration and the second four were $2 \mathrm{~min}$ in duration. All eight lesions had a $100 \mu \mathrm{A}$ current.

\section{Deaf}

Birds were deeply anesthetized with Equithesin $(0.04 \mathrm{cc})$, and then secured to an adjustable surgical platform. A small incision was made in the skin covering the outer surface of the external meatus. The tympanic membrane was exposed, pierced, and retracted. Within the middle ear, the extracolumella was disconnected from the columella. The footplate is bound to the dorsal end of the columella and both were removed by extraction with fine forceps. Briefly, the columella can be grasped with forceps by the stem that protrudes from the oval window. With the opening to the oval window cleared, a fine wire hook was used to extract the cochlea. The extracted cochlea was examined under a microscope to verify that it had been removed in its entirety. After surgery, the incision was treated with an antiseptic and sealed with veterinary adhesive, and the bird was returned to its home cage.

\section{Control surgery}

For HVC and LMAN control surgeries (HVCcs; LMANcs), birds were deeply anesthetized and received a scalp incision and removal of a small cranial plate over the location of the respective nuclei for which it was a control. After surgery, the incision was treated with an antiseptic and sealed with veterinary adhesive, and the bird was returned to its home cage. We did not include an HVCml + Deafcs group because standard deafening controls are typically either no surgery (Nordeen and Nordeen, 1992; Brainard and Doupe, 2000, 2001; Lombardino and Nottebohm, 2000; Watanabe et al., 2006) or control surgery up to the point of cochlea removal (Wang et al., 1999; Lombardino and Nottebohm, 2000) and results from both control methods do not differ (Wang et al., 1999). Therefore, birds in the HVCml + LMANcs group were sufficient to control for the nonspecific effects of a second surgery after HVC microlesions.

\section{Confirmation of lesions}

At the end of the 3 week postoperative period, birds were given an overdose of Equithesin $(0.08 \mathrm{cc})$ and intracardially perfused with saline followed by ice-cold $4 \%$ paraformaldehyde. After overnight postfix by immersion in $4 \%$ paraformaldehyde, brains were sectioned coronally on a vibratome at $40 \mu \mathrm{m}$ thickness. Serial sections were then mounted onto microscope slides, thionin stained, and coverslipped. The procedures used for lesion confirmation (both HVC microlesions and LMAN ablation) were similar to those described by Thompson and Johnson (2007).

\section{HVC}

Nissl-stained serial sections of caudal telencephalon that contained HVC were examined using a digital camera attached to a light microscope at low power $(5 \times$ objective). For each bird that received HVC microlesions, Neurolucida software (MicroBrightField, Colchester, VT) was used to trace and estimate the volume of the microlesion and the volume of remaining HVC. This was done separately for left and right HVC.

Percent HVC remaining. Two methods were used to estimate percent HVC remaining. Both methods used the same equation: (total HVC volume - microlesion volume)/total HVC volume. However, the two methods differed in the derivation of total HVC volume. In one method, we generated a "reconstructed" total HVC volume by adding the volume of microlesion to the volume of remaining (unlesioned) HVC. To trace and estimate the volume of the microlesion, we included only lesion damage that intersected the dorsal or ventral borders of HVC (borders that were clearly identifiable in the coronal plane of section). When present, lesion damage lateral to HVC was not included in the tracings because such lesion damage distorted the lateral border of HVC. Similarly, when tracing and estimating the volume of remaining $\mathrm{HVC}$ in sections with microlesion damage, we assumed the lateral edge of HVC adjacent to any lesion damage to be the actual lateral border of HVC.

In the second method, we calculated the percent $\mathrm{HVC}$ remaining in each bird using a total HVC volume that was an average unilateral volume of intact HVC for adult male zebra finches $\left(0.32 \mathrm{~mm}^{3}\right.$; derived from $n=6$ birds that did not receive HVC microlesions). Although this method will not account for individual differences in HVC volume, it has the advantage of removing the uncertainty associated with tracing HVC borders in lesion-damaged tissue.

Percentage of sections with HVC damage. For each bird with HVC microlesions, we further quantified the bilateral extent of microlesion damage by summing the number of sections of HVC that contained microlesion damage and dividing by the total number of sections that contained HVC. This was also done separately for left and right HVC.

\section{LMAN}

Photomicrographs of Nissl-stained, bilateral, serial sections through the rostral telencephalon were imported into Image-Pro 3.0 (Media Cybernetics, Silver Spring, MD). Analysis tools incorporated into the ImagePro program generated area estimates for serially traced contours of LMAN.

Percent LMAN remaining. Nonablated regions (i.e., area remaining) of LMAN, defined by Nissl stain, were traced, and the areas were summed 
A Bird 610
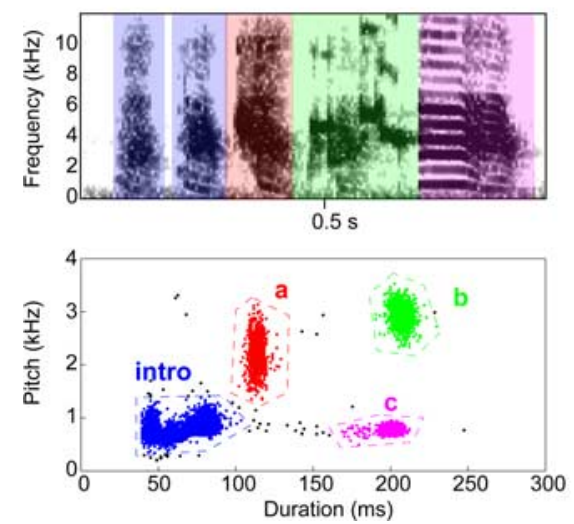

Figure 2. Note types in the spectrograph and note clusters in the scatter plot are color-matched to demonstrate how notes were identified for note transition probabilities. $\boldsymbol{A}$, Bird 610 had three motif notes $(a, b$, and $c)$ and two intro note variants. Although notes $b$ and $c$ had similar durations, their corresponding clusters in the scatter plot were easily distinguished by the difference in their pitch. Similarly note $a$ was distinguished from the intro notes by both duration and pitch. $\boldsymbol{B}$, Pitch goodness generated the most distinct clusters for bird 628 and thus was the feature used to identify notes. Notes $b$ and $c$ share similar durations but were easily differentiated by their pitch goodness. Likewise, note $d$ was only distinguished from intro notes by its pitch goodness; however, note $a$ was set apart by its duration and pitch. A small cluster of black dots outside the note boundaries is indicated by "call" and represented an infrequent call produced during singing.

and multiplied by section thickness. The remaining LMAN volume from each side was then divided by an average volume for intact unilateral LMAN $\left(0.11 \mathrm{~mm}^{3}\right.$; obtained from five intact birds) to estimate a percent unilateral LMAN remaining. Both unilateral estimates of remaining LMAN were then averaged to derive a value for total percent bilateral LMAN remaining.

\section{Recording protocol}

Birds were maintained in complete social isolation for the duration of the experiment. Therefore, only "undirected" songs (i.e., not directed toward a female) were recorded and analyzed. Although there is a normal degree of variability that characterizes undirected song (Sossinka and Bohner, 1980; Kao et al., 2005), it is far less than the variability we report here after HVC microlesions.

For all birds, song production was recorded in $24 \mathrm{~h}$ blocks using a unidirectional microphone fastened to the side of the internal cage. Sounds transmitted by the microphone were monitored through a computer that was running sound-event triggered software (Avisoft Recorder; Avisoft Bioacoustics, Berlin, Germany). Song was captured in bouts (2-7 s bursts of continuous singing during which the motif may be repeated one to five times) and each song bout was saved as a time-stamped .wav file ( $44 \mathrm{kHz}$ sampling rate) onto the computer hard drive where each day of singing by each bird was saved under a single directory.

Three preoperative days of singing were obtained after the 2 week acclimation period. Postoperative singing was monitored every day beginning with the first day after the first surgery up to the day of overdose with anesthetic. Birds took on average $3 \mathrm{~d}$ ( $\pm 1 \mathrm{~d}$; SEM) to begin singing after the first surgery and after the second surgery birds took an average of $2 \mathrm{~d}( \pm 1 \mathrm{~d}$; SEM) before they resumed singing. To insure that we captured all preoperative and postoperative song bouts, triggering settings for Avisoft Recorder were biased in favor of false-positive captures (i.e., .wav files composed of repeated calls and/or cage noises). This was particularly important for accurate capture of song bouts after HVC microlesions, in which song destabilization persists for several days. Therefore, for each bird and on each day of preoperative and postoperative singing, falsepositive sound files were selectively deleted from each bird's database of .wav files.

Detection and deletion of false-positive .wav files involved a three-step process. First, we used Spectrogram (version 13.0; Visualization Software LLC) to convert sound files (.wav) into frequency spectrograph image files (.jpg). Second, we used an image-management program that contains an image-pattern recognition module (IMatch; M. Westphal,
Usingen, Germany) to identify .jpg files that contained singing-related spectra and deleted those images from the .jpg database. For the third and final step, we converted the file names of remaining false-positive images (i.e., .jpg files that did not contain singing-related spectra) from .jpg back to .wav, and then batch-deleted those files from the main database of .wav files. Thus, each day of singing by each bird was reduced to a directory that contained only .wav files with song bouts. Although each of these .wav files contained a song bout, some files also included cage noises (pecking on the cage floor, wing flaps, or beak-wiping on the perch) and short and long calls that birds often produce in close temporal proximity to a song bout.

\section{Analysis of song behavior}

As described above, we allowed $1 \mathrm{~d}$ of postoperative vocalization after the first surgery, which then served as a basis for behavioral comparison between groups with different manipulations, and between the two manipulations within a particular group. After the second surgery, all birds had 3 weeks of postoperative recovery. Selection of a 3 week postoperative period was based on previous work showing that recovery of stereotyped song after HVC microlesions is typically achieved within 1 week (Thompson and Johnson, 2007). Because all birds in the present study experienced two surgeries, we extended the recovery period to 3 weeks rather than 2 weeks (Thompson and Johnson, 2007) in the event that some birds were slow to recover. However, on visual and auditory inspection of song behavior from birds in all experimental groups, it was clear that song stabilized within 1 week after the second surgery. Therefore, for statistical analysis, we selected the first $3 \mathrm{~d}$ of preoperative singing (Pre1, Pre2, and Pre3), the first day of singing after the first surgery (Post 1 ), the next $7 \mathrm{~d}$ of singing after the second surgery (Post2-8), and the final day of singing before killing (Final). This resulted in analysis of $12 \mathrm{~d}$ of singing from each bird in the study.

\section{Production analysis}

To measure the recovery of daily song bout production after surgery, for each bird in all groups we calculated the average daily number of song bouts across the 3 preoperative recording days (Pre1-3). The average of the 3 preoperative days was used to establish a baseline for daily bout production for each bird. To quantify the recovery of song bout production, the number of bouts produced on Post1-8 and Final were divided by the preoperative baseline to calculate a percent baseline of song bout production.

\section{Phonology analysis (note measurement)}

For each bird, we measured phonology using the first 300 song bouts produced on each of the $12 \mathrm{~d}$ selected for statistical analysis (Pre1-3, Post $1-8$, and Final). This resulted in the analysis of $\sim 5000$ notes per bird per day [motif note number was similar across all groups $($ mean \pm SEM $):$ HVCml + Deaf, $3.6 \pm 0.51$; HVCcs + Deaf, $4.5 \pm$ 0.43; HVCml + LMAN Ablation, $4.17 \pm 0.17$; HVCcs + LMAN Ablation, $4.6 \pm 0.75$; and $\mathrm{HVCml}+\mathrm{LMANcs}, 3.67 \pm 0.33]$. However, if a bird produced $<300$ bouts on a given day (common in all birds during initial postoperative days), all song bouts produced during that day were analyzed.

We quantified each bird's song by converting each day of singing (Pre1-3, Post1-8, Final) into a data set in which each sound (note) was isolated and measured for duration and four independent spectral features (pitch, FM, entropy, pitch goodness). This was accomplished using the Feature Batch module in the Sound Analysis Pro (SA+) software (version 1.04) (Tchernichovski et al., 2000). Briefly, syllable-threshold controls in SA+ (amplitude, entropy, minimum syllable, and gap duration) were manually configured for the song of each individual bird so 
that the software reliably identified each note in the bird's motif. Initially, 20-40 randomly selected song bouts from Prel were used to identify threshold settings for each bird. Scatter plots were then used to verify that threshold settings had been accurately specified; for these plots, note duration was always plotted on the $x$-axis and one of the measured spectral features (pitch, FM, entropy, pitch goodness) was plotted on the $y$-axis. Within these plots, each data point represents an individual note and thus discrete clusters of data points signify repeated production of a specific note type. Finally, clusters observed within the scatter plots were compared against a frequency spectrograph of the bird's motif to confirm that individual notes were represented by discrete clusters of data points (for examples, see Fig. 2). After this process, threshold settings for each bird were noted and then applied without change to all song bouts produced in Pre2-3, Post1-8, and Final, insuring valid preoperative and postoperative comparisons.

\section{Phonology analysis (Kullback-Leibler distance)}

Each day of singing by each bird resulted in a data sheet that contained values for the duration, pitch, FM, entropy, and pitch goodness of each note. For each bout analyzed, these values were listed in the sequence that the notes were produced. The data sheets were then used to generate four scatter plots that described each day of singing (duration vs pitch, duration vs FM, duration vs entropy, duration vs pitch goodness). To assess vocal change in each bird, the feature scatter plots generated from a baseline day of singing (Pre1) were compared with those generated on succeeding singing days (Pre2-3, Post1-8, and Final) using the Kullback-Leibler (K-L) distance, described below.

Let $P_{1}, P_{2}, P_{3}$ represent the two-dimensional scatter plots for one pair of features (e.g., duration vs entropy) generated during the three preoperative singing days (Pre1-3), respectively. Also, let $P_{4}, P_{5}, \ldots, P_{12}$ represent the scatter plots in the nine postoperative singing days (Post1-8 and Final), respectively. To quantify the rate of recovery of the birdsong after surgeries we estimated the recovery of $P_{k}$ toward $P_{1}$, where $k=4,5$, $\ldots, 12$. As a baseline criterion to quantify the normal day-to-day variation in phonology, we also estimated the "recovery" of $P_{2}$ and $P_{3}$ toward $P_{1}$. The tool that we used for these analyses, the $\mathrm{K}-\mathrm{L}$ distance, is a standard information-theoretic measure for the dissimilarity of two patterns (Kullback and Leibler, 1951). In this case, the patterns are the scatter plots obtained during the 12 recording sessions (days).

To use the $\mathrm{K}-\mathrm{L}$ distance to measure the recovery rate, a procedure was developed for estimating the two-dimensional distribution of the data points in each of the recording sessions. We partitioned the twodimensional scatter plot into an $M$ by $N$ array of bins (note duration was partitioned into $M$ equally spaced bins, and a second feature was partitioned into $N$ equally spaced bins). Thus, we have $M \times N$ twodimensional bins. To have an appropriate and consistent estimation of the distribution, $M$ and $N$ were chosen as a constant value, 15 , over all the data recordings. The probability distribution in each session was estimated by simply counting the number of data points in each bin and dividing by the total number of data points in the session.

Let $Q_{1}, Q_{2}$, and $Q_{3}$ denote the estimated two-dimensional probability distributions in the 3 preoperative days, respectively, and $Q_{4}, Q_{5}, \ldots, Q_{12}$ denote the distributions in the 9 postoperative days, respectively. For $k=$ $1,2, \ldots, 12$, the $\mathrm{K}-\mathrm{L}$ distance (measured in units of bits) between $Q_{k}$ and $Q_{1}$ was defined as follows:

$$
D_{\mathrm{KL}}\left(Q_{1} \| Q_{k}\right)=\sum_{m=1}^{M} \sum_{n=1}^{N} q_{1}(m, n) \log _{2} \frac{q_{1}(m, n)}{q_{k}(m, n)},
$$

where $q_{1}(m, n)$ and $q_{k}(m, n)$ were the estimated probabilities for bin ( $m$, $n$ ) for PRE1 and the $k$ th session, respectively. The K-L distance describes

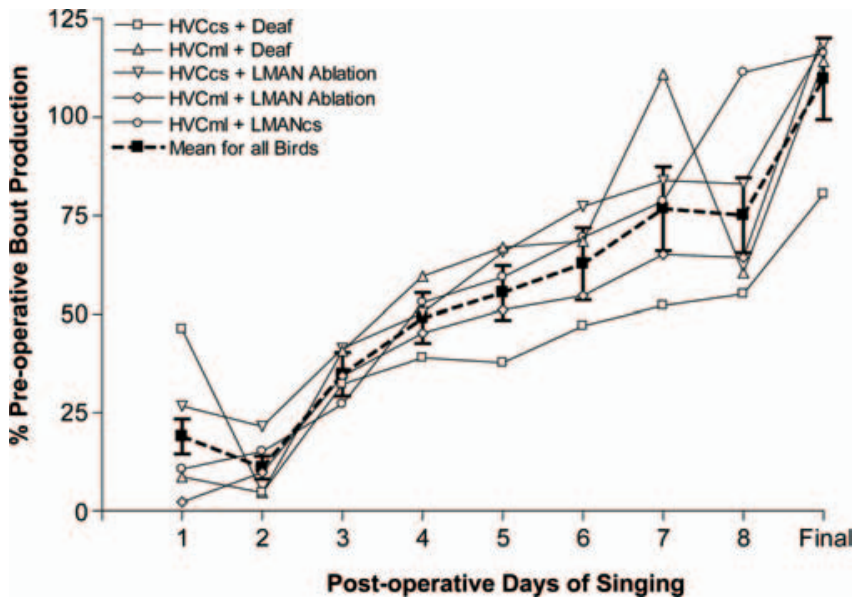

Figure 3. Recovery of bout production across postoperative days of singing. The $x$-axis represents days of postoperative singing: 1 indicates the first day of postoperative singing after the first surgery, 2-8 indicates the first $7 \mathrm{~d}$ of postoperative singing after the second surgery, and Final represents the last day of singing (3 weeks after second surgery). Error bars indicate SEM.

the "distance" between two distributions, with $D_{\mathrm{KL}}\left(Q_{1} \| Q_{k}\right) \geq 0$ for any $Q_{1}$ and $Q_{k}$, and $D_{\mathrm{KL}}\left(Q_{1} \| Q_{k}\right)=0$ if and only if $Q_{1}$ and $Q_{k}$ are the same distributions.

Quantifying rate of postoperative vocal recovery $(\tau)$. To calculate the relative rate of recovery for each of the four features in the postoperative sessions, we first normalized $D_{\mathrm{KL}}$ so that $D_{\mathrm{KL}}=1$ at Post1. We then fit each normalized curve with an exponential decay function: $n D_{k}=\exp (-(k-4) / \tau)$, where $n D_{k}$ denotes the normalized $\mathrm{K}-\mathrm{L}$ distance at the $k$ th session, $k=4, \ldots, 12$, and $\tau$ denotes the recovery rate (in units of days). A large $\tau$ implies a slow recovery, whereas a small $\tau$ implies a fast recovery. The $\tau$ values were used for statistical comparison of the rate of vocal recovery for HVCml + LMAN Ablation and HVCml + LMANcs groups.

Quantifying postoperative vocal stability ( $\delta$ ). Postoperative phonology for HVCml + Deaf and HVCcs + Deaf groups was relatively stable, so the $D_{\mathrm{KL}}$ values in the postoperative sessions remained relatively constant. Therefore, instead of estimating recovery rate, we computed a different statistic, $\delta$, which describes the difference in the time-averaged $\mathrm{K}-\mathrm{L}$ distance before and after the first surgery. That is,

$$
\delta=\frac{1}{9} \sum_{k=4}^{12} D_{\mathrm{KL}}\left(Q_{1} \| Q_{k}\right)-\frac{1}{2} \sum_{k=2}^{3} D_{\mathrm{KL}}\left(Q_{1} \| Q_{k}\right)
$$

The size of $\delta$ reflects the extent of preoperative versus postoperative change in the scatter plots, with a large $\delta$ indicating that postoperative phonology is highly dissimilar from preoperative phonology. 
A HVCcs + Deaf (Bird 621)

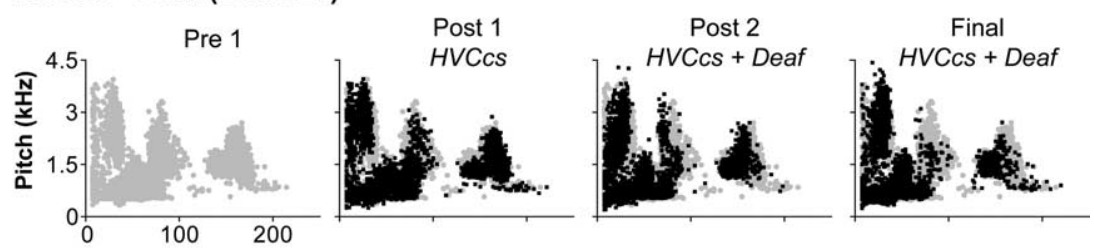

\section{B $\mathrm{HVCml}+$ Deaf (Bird 617)}

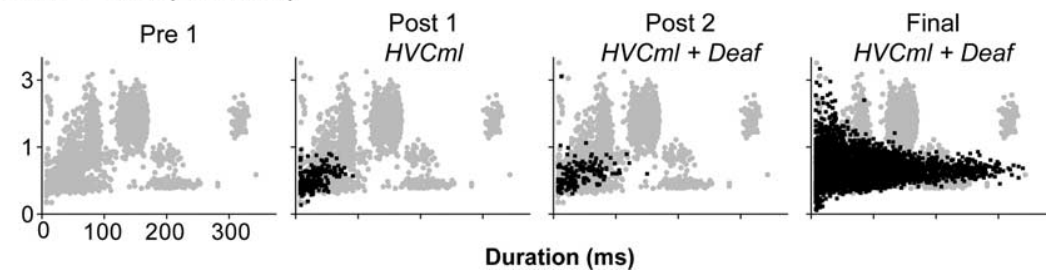

Figure 4. Duration versus pitch scatter plots for representative birds from HVCcs + Deaf and HVCml + Deaf groups (the gray dots represent preoperative notes, and the black dots indicate postoperative notes). $\boldsymbol{A}$, Control surgery and deafening had little effect on phonology. $\boldsymbol{B}$, HVC microlesions caused the production of notes that were relatively limited in pitch and duration (Post1) that continued after deafening (Post2). However, by the end of recovery, there was an increase in the phonological range of destabilized note types (Final).

\section{A $\mathrm{HVCml}+$ Deaf}

$$
\text { Pre1 }
$$

Post $1(\mathrm{HVCml})$
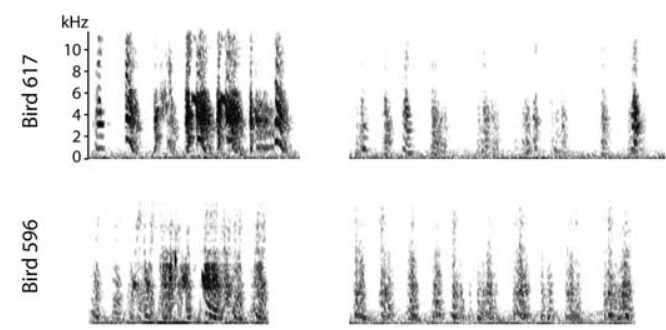

Post Final (Deaf)

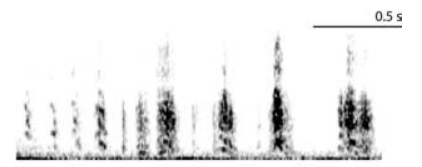

\section{B HVCmI + LMAN Ablation}

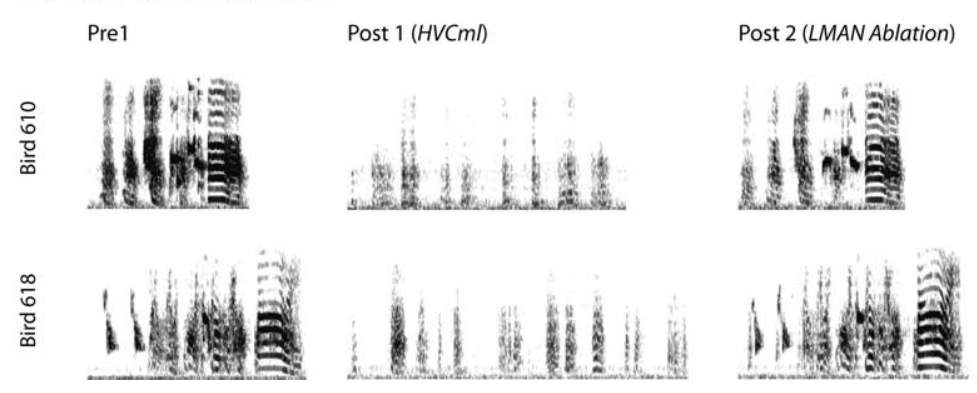

Figure 5. Frequency spectrographs from two representative birds from $\mathrm{HVCml}+$ Deaf and $\mathrm{HVCml}+\mathrm{LMAN}$ Ablation groups. $\boldsymbol{A}$, Loss of auditory feedback after HVC microlesions prevented recovery of the preoperative motif. $\boldsymbol{B}$, LMAN ablation subsequent to HVC microlesions induced sudden recovery of the preoperative motif. The Post2 spectrographs for bird 610 and bird 618 are motifs from the first bout produced after LMAN ablation.

the sequence analysis, we selected the duration versus feature scatter plot with the greatest number of distinct note clusters. That is, although there were never more note clusters than notes in a bird's motif, some duration versus feature scatter plots produced fewer note clusters than actual notes in a bird's motif. For example, if a bird's motif contained five notes, the duration versus pitch goodness scatter plot may have five distinct clusters, whereas only four clusters may be apparent in the duration versus FM plot because of cluster overlap; this would occur if two or more of the motif notes shared a similar duration and FM, but differed in pitch goodness. We would then use the duration versus pitch goodness scatter plot for the sequence analysis in this bird.

A polygonal boundary was traced around each note cluster in the Prel scatter plot to define the range of temporal and spectral values typical of each note type in the motif (for examples, see Fig. 2). These note boundaries were used to identify note types produced on each subsequent day of singing. Any point that fell outside of the note boundaries was defined as note type $n$, or nonmotif notes (this included occasional call notes that occurred within a bout). Introductory notes in a bout were classified as "intro."

For each day of singing, we obtained the temporal ordering of note types within, but not between bouts, thus omitting transitions between bouts. We then computed the number of transitions between note types $a$ and $b$, or $a$ and $c$, etc. We denote these as $N_{a b}, N_{a c}$, etc. Using $N_{\text {tot }}$ to denote the total number of transitions made within bouts, the estimated transition probabilities are $T_{a b}=N_{a b} / N_{\text {tot }}$, $T_{a c}=N_{a c} / N_{\text {tot }}$, etc. It was found that most of the transition probabilities were very small. To focus on the major transitions, we grouped all transition types with probability $<0.05$ as a single type, denoted as "others."

We refer to the transitions that occur between notes in a motif as "motif transitions." For each recording session and each bird, the motif transition probabilities were summed, and then the sums were averaged across the population of birds in a particular group to obtain the "average probability of motif transitions." This was computed for Pre1, Post1, Post2, and Final, and used as a measure of the perturbation away from and recovery of the correct note sequence after the surgical manipulations.

\section{Statistics}

\section{Sequence analysis}

Data sheets generated for the phonology analysis were organized so that both bouts and notes were listed in the order that they were produced. These bout and note order data were used to assess the recovery of note sequencing in HVCml + LMAN Ablation and HVCml + LMANcs birds (these were the only groups to show vocal destabilization and subsequent recovery).

For each individual bird, note sequencing was accomplished by first examining a frequency spectrograph of the bird's motif at Pre1 and comparing it with each of the four duration versus feature scatter plots generated at Pre1. Each note within a motif tended to fall within a cluster in a scatter plot (for color-coded examples, see Fig. 2). For
All analyses were performed using SigmaStat 2.03 (SPSS, Chicago, IL) and Matlab (MathWorks, Natick, MA). Two-way ANOVAs were used to evaluate group differences in LMAN and HVC morphology (group by hemisphere) and bout production (group by day). One-way ANOVAs were used to assess group differences in $\delta$ and $\tau$, and within-group differences in preoperative versus postoperative $\mathrm{K}-\mathrm{L}$ distance values. Oneway ANOVAs were also used to analyze preoperative versus postoperative change in motif note transition probabilities. All pairwise comparisons of statistically significant $(p<0.05)$ main effects or interactions were evaluated using Student-Newman-Keuls (SNK) tests. 


\section{A HVCcs + Deaf}

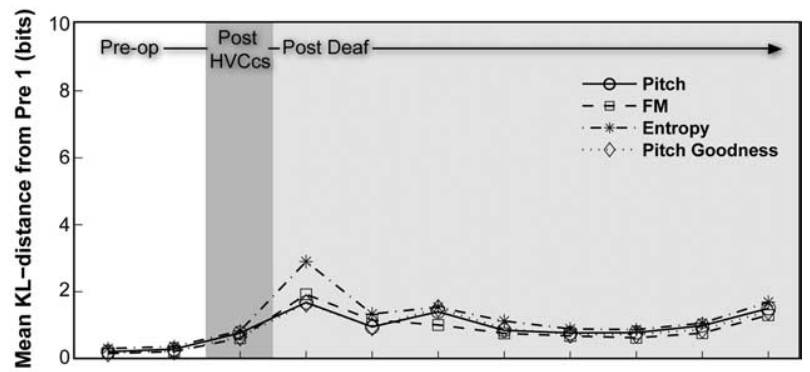

\section{B $\mathrm{HVCml}+$ Deaf}

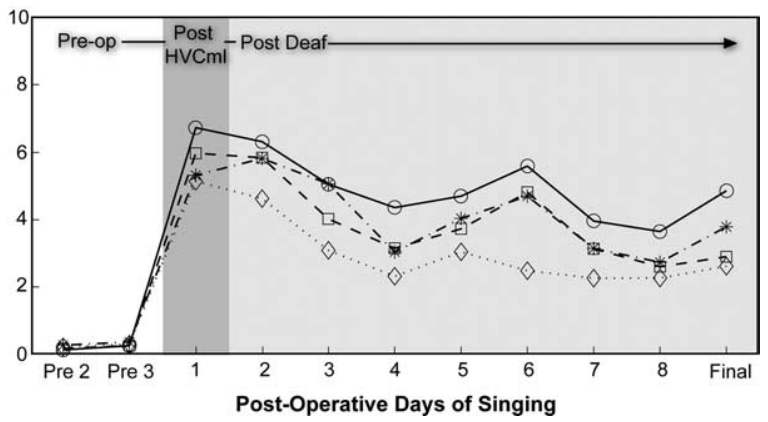

Figure 6. Mean K-L distance values from Pre1 describe changes in postoperative phonology for birds in the HVCcs + Deaf and HVCml + Deaf groups. Preoperative comparisons (Pre2 and Pre3) show very little variation in phonology. $\boldsymbol{A}$, HVC control surgery followed by deafening caused a slight change to phonology. $\boldsymbol{B}, \mathrm{HVC}$ microlesions induced a marked increase in phonological dissimilarity that was sustained by the loss of auditory feedback.

\section{Results}

\section{Microlesion and ablation morphometry}

Table 1 shows a summary of HVC and LMAN lesion damage that includes percent sections with HVC damage, percent HVC remaining, and percent LMAN remaining.

\section{Sections with HVC damage}

Statistical analysis revealed that groups did not differ in the amount of HVC damage (main effect of group, $F_{(2,33)}=0.28 ; p=$ 0.76 ) and that the amount of damage was bilaterally symmetrical (main effect of hemisphere, $F_{(1,33)}=0.16 ; p=0.70$ ).

\section{Percent HVC remaining}

Two different methods were used to assess the bilateral symmetry of HVC microlesions across groups. Results from the first method (i.e., total HVC volume derived from anatomical reconstruction of HVC) revealed no bilateral difference (main effect of hemisphere, $\left.F_{(1,33)}=0.01 ; p=0.94\right)$ nor any group difference (main effect of group, $F_{(2,33)}=1.53 ; p=0.23$ ) in percent $\mathrm{HVC}$ remaining.

Analysis of results from the second method (i.e., total HVC volume derived from an average unilateral volume of intact HVC) revealed no bilateral difference in percent HVC remaining $\left(F_{(1,33)}=0.16 ; p=0.69\right)$. However, a significant group difference was observed (main effect for group, $F_{(2,33)}=4.90 ; p<0.05$ ). Pairwise comparisons showed that HVCml + LMAN Ablation birds had less remaining HVC than HVCml + Deaf birds (no other comparisons were significant) (Table 1). This difference does not alter the interpretation of behavioral findings (described below) because the vocal behavior of these two groups is never compared statistically; in this regard, it is important to note that

\section{A HVCcs + Deaf Birds}

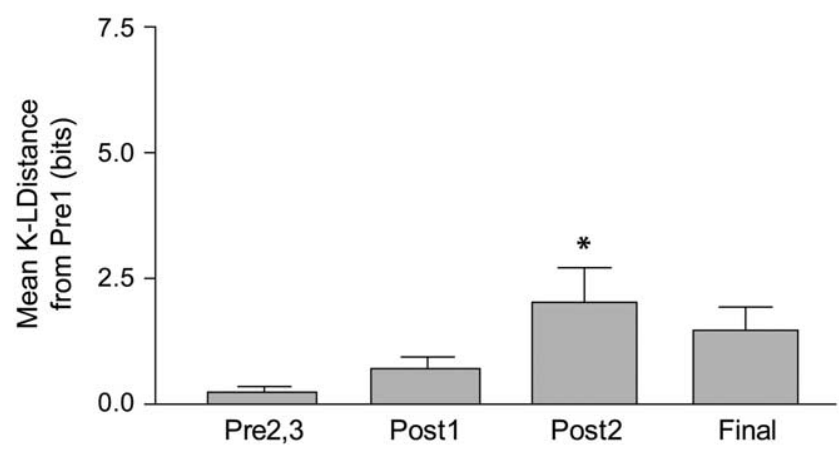

\section{B $\mathrm{HVCml}+$ Deaf Birds}

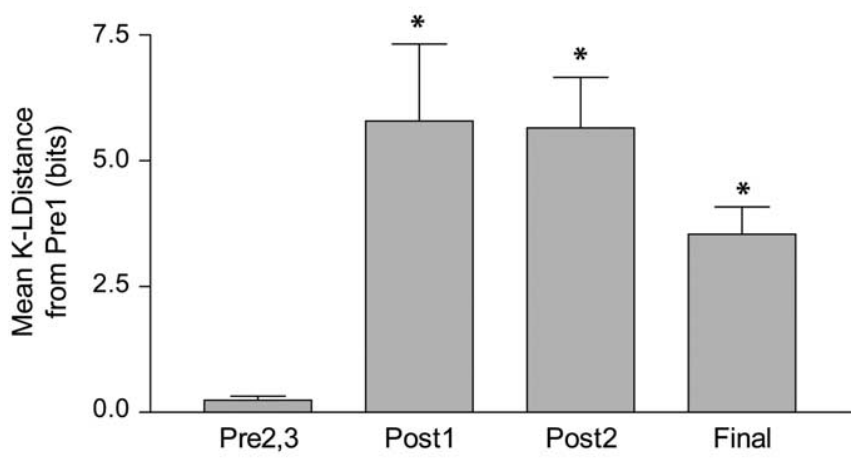

\section{HVCcs + LMAN Ablation Birds}

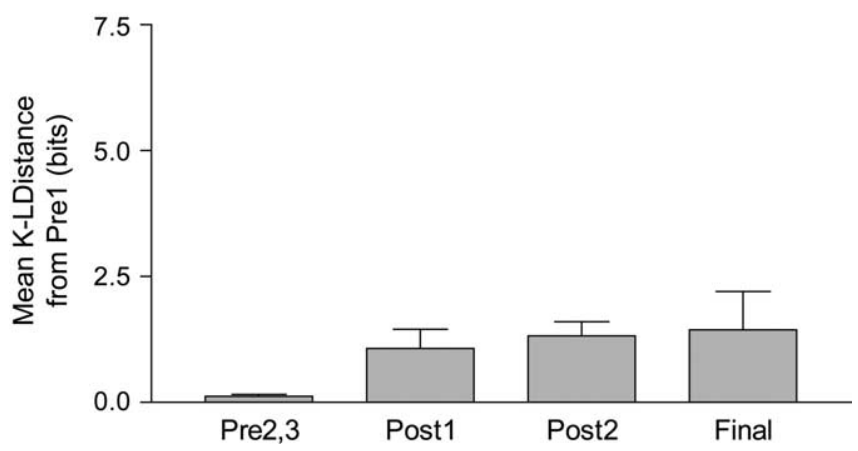

Figure 7. Mean $\mathrm{K}-\mathrm{L}$ distance values describe acute and prolonged change in phonology for birds in the HVCcs + Deaf, HVCml + Deaf, and HVCcs + LMAN Ablation groups. *Significant difference from Pre2,3 $(p<0.05)$. A, Deafening (Post2) but not HVC control surgery (Post1) was associated with a significant change in phonology when compared with preoperative days of singing (Pre2,3). However, this effect was acute and did not persist to the end of the recovery period (Final). B, HVC microlesions produced a significant change in phonology (Post1) that persisted after deafening (Post2) and the 3 week recovery period (Final). Mean $\mathrm{K}-\mathrm{L}$ distance values for the 3 postoperative days of singing (Post1, Post2, and Final) were not different from each other. C, Phonology showed no significant change after HVC control surgery (Post1), LMAN ablation (Post2), or the 3 week recovery period (Final). Error bars indicate SEM.

percent HVC remaining did not differ between HVCml + LMAN Ablation and HVCcs + LMAN Ablation birds.

\section{Percent LMAN remaining}

LMAN lesions were similar in HVCml + LMAN Ablation and HVCcs + LMAN Ablation groups. That is, the two groups did not differ in percent remaining LMAN (main effect of group, $\left.F_{(1,10)}=0.54 ; p=0.48\right)$. Table 1 also indicates that percent LMAN remaining (in both groups) was on average close to $7 \%$. 
A HVCml + LMANcs (Bird 628)

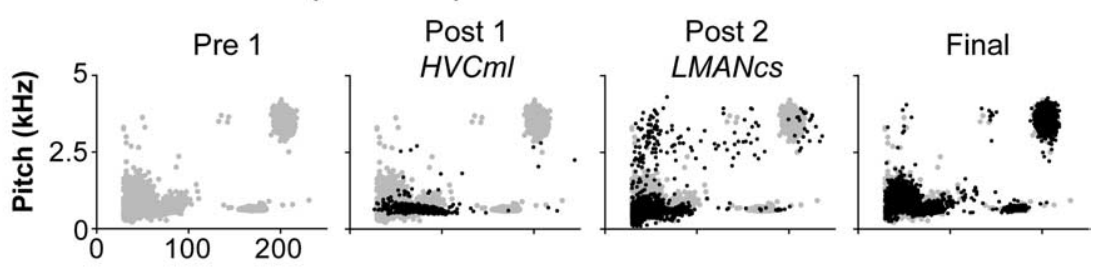

B HVCml + LMAN Ablation (Bird 610)

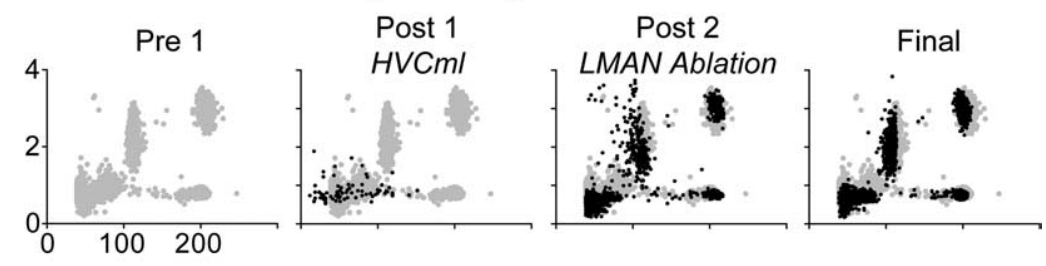

\section{HVCcs + LMAN Ablation (Bird 629)}

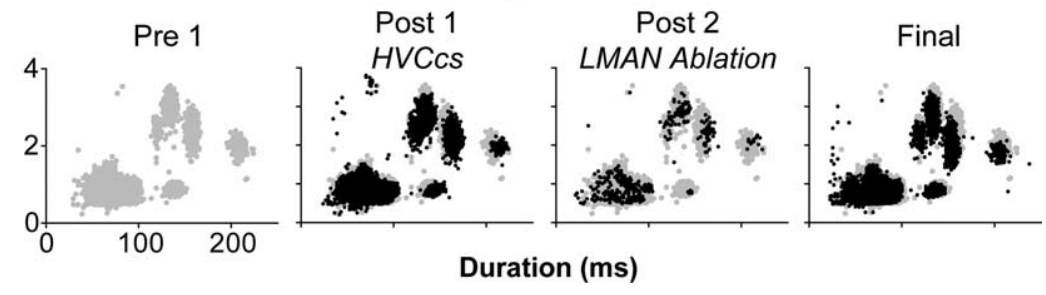

Figure 8. Duration versus pitch scatter plots for representative birds from $\mathrm{HVCml}+\mathrm{LMANCs}, \mathrm{HVCml}+\mathrm{LMAN}$ Ablation, and HVCcs + LMAN Ablation groups (the gray dots represent preoperative notes, and the black dots indicate postoperative notes). $\boldsymbol{A}_{\text {, }}$ HVC microlesions followed by control surgery caused the production of destabilized notes that were limited in pitch and duration (Post1 and Post2); however, by the end of the recovery period preoperative phonology was restored (Final). B, LMAN ablation restored preoperative phonology at Post2, although a few dissimilar notes were still produced. $\boldsymbol{C}$, Control surgery and LMAN ablation had little effect on phonology.

From these birds, the largest percent LMAN remaining was $27 \%$. Kao et al. (2005) found that $>70 \%$ ablation of LMAN was sufficient to abolish the behavioral influence of LMAN in adult zebra finches.

\section{Postoperative recovery of bout production}

Regardless of surgery manipulation singing was reduced on Post1 (first day of singing after first surgery) and Post2 (first day of singing after second surgery), with a gradual recovery of daily bout production over the 3 week postoperative period (Fig. 3). Although singing was reduced on Post1 and Post2, all birds with HVC microlesions produced a sufficient number of song bouts $(42 \pm 16$; mean \pm SEM $)$ to characterize phonology and note sequence.

Statistical analysis of bout production data (group by day) revealed that there was a significant effect of day $\left(F_{(8,251)}=15.15\right.$; $p<0.001)$. However, singing did not vary by group $\left(F_{(4,251)}=\right.$ $1.91 ; p=0.11)$ nor was there an interaction of group by day $\left(F_{(32,251)}=0.59 ; p=0.96\right)$. Because the recovery of bout production was similar across all experimental and control groups, differences in the recovery of phonology and note sequence are not attributable to group differences in bout production.

Vocal recovery requires auditory feedback

Birds deafened after HVC microlesions were unable to recover their preoperative song. This can be seen in Figure 4 in which duration versus pitch scatter plots of phonology from two representative birds show the effects of deafening after HVC control surgery (HVCcs + Deaf) or after HVC microlesions (HVCml + Deaf). We selected duration versus pitch scatter plots to visualize phonology based on evidence that birds actively tune their vocal tract to the fundamental frequencies of their song (Riede et al., 2006). However, scatter plots using the other spectral features (duration vs FM, entropy, or pitch goodness) always produced patterns that were similar to the duration versus pitch scatter plots.

Figure $4 A$ shows that HVC control surgery (Post1) and deafening (Post2-Final) induced modest change in the phonology of some notes (i.e., black postoperative note clusters primarily localized over gray preoperative note clusters). In contrast, Figure $4 B$ shows that HVC microlesions (Post1) caused the production of notes that all fell into the bottom left corner of the scatter plot (i.e., all notes were limited in duration and pitch). After deafening (Post2-Final) the production of pitchlimited notes continued, although by the end of the recovery period (Final) the duration of these notes became highly variable. Figure $5 A$ shows example spectrographs from two representative birds in the HVCml + Deaf group.

We used the $\mathrm{K}-\mathrm{L}$ distance as a measure of the difference in the scatter plots on different days of singing (see Materials and Methods). A small $\mathrm{K}-\mathrm{L}$ distance indicates that the scatter plots are similar. Figure 6 shows the four spectral features used for $\mathrm{K}-\mathrm{L}$ distance analysis (pitch, FM, entropy, and pitch goodness) in HVCcs + Deaf and HVCml + Deaf birds. When compared with Pre1, K-L distance values for Pre2 and Pre3 were low in both groups, indicative of highly stable phonology across preoperative days of singing.

Figure $6 A$ shows that HVCcs + Deaf birds produced small changes in phonology after HVC control surgery (Post1) and deafening (Post2) that persisted through the final day of recovery. Statistical analysis of mean $\mathrm{K}-\mathrm{L}$ distance values revealed that this change was significant $\left(F_{(3,23)}=3.38 ; p<0.05\right)$, but only at Post2 (Fig. 7A). Previous literature suggests that song change after the loss of auditory feedback in adult zebra finches requires 1-4 months (Nordeen and Nordeen, 1992; Leonardo and Konishi, 1999; Lombardino and Nottebohm, 2000; Brainard and Doupe, 2000), although delayed auditory feedback can produce immediate effects on song (Cynx and von Rad, 2001). Therefore, the significant change in phonology observed at Post 2 could indicate an acute, transient effect of deafening. However, because control surgery alone produced small changes in phonology (Fig. 7 A, C, Post1), we cannot rule out the possibility that nonspecific effects of the surgical manipulations contributed to this small but significant change in phonology after deafening.

Figure $6 B$ shows that HVCml + Deaf birds produced large changes in phonology after HVC microlesions (Post1) that was then sustained in the absence of auditory feedback during the entire period of recovery (Post2-Final). Statistical analysis of mean K-L distance values revealed that these changes in phonology were significant at Post1, Post2, and Final $\left(F_{(3,19)}=7.30 ; p<0.05\right)$ (Fig. $\left.7 B\right)$. 
Thus, when deprived of auditory feedback, birds with HVC microlesions showed no evidence of vocal recovery.

To statistically compare the postoperative vocal behavior of the two deafening groups (HVCcs + Deaf and HVCml + Deaf) we used $\delta$, defined as the difference between the time-averaged $\mathrm{K}-\mathrm{L}$ distance before (Pre2 and Pre3) and after the first surgery (Post1-8, Final). $\delta$ is expressed in units of bits (see Materials and Methods). For each bird, $\delta$ was calculated for each of the four spectral features (pitch, FM, entropy, and pitch goodness). Statistical analysis of $\delta$ values for each bird revealed that HVCml + Deaf birds $(\delta \pm \mathrm{SEM}=3.83 \pm 0.37$ bits $)$ had a significantly larger postoperative change in phonology than HVCcs + Deaf birds $(\delta \pm$ SEM, $0.87 \pm$ 0.04 bits; $\left.F_{(1,43)}=78.36 ; p<0.001\right)$. Thus, deafening produced a substantial and sustained increase in phonological dissimilarity only when preceded by HVC microlesions.

\section{Sudden vocal recovery after LMAN ablation: phonology}

Birds with HVC microlesions showed a sudden recovery of their motif after LMAN ablation. That is, three of six birds produced their preoperative motif in the first bout produced on Post2 (Fig. $5 B$ ) and all HVCml + LMAN Ablation birds produced their preoperative motif during Post2. Figure 8 shows duration versus pitch scatter plots of phonology for representative birds from HVCml + LMANcs, HVCml + LMAN Ablation, and HVCcs + LMAN Ablation groups. Scatter plots for birds that received HVC microlesions (Fig. 8A,B) show that Post1 singing consisted of notes characterized by low pitch and duration (compare with spectrographs for Post 1 in Fig. $5 B$ ). However, scatter plots from Post 2 singing show that LMAN ablation but not control surgery facilitated recovery of notes that resembled preoperative note types (compare Post2 in Fig. 8A,B). Importantly, LMAN ablation did not induce a dramatic change in phonology when it was preceded by control HVC surgery (Fig. $8 C$ ). The slight offset in note clustering seen at Final in birds with LMAN ablation (Fig. $8 B, C)$ may reflect the influence of LMAN ablation on note variation (Kao et al., 2005; Kao and Brainard, 2006).

Figure 9 shows group data for the four spectral features used for $\mathrm{K}-\mathrm{L}$ distance analysis (pitch, FM, entropy, and pitch goodness). Figure 9, $A$ and $B$, shows that there was a dramatic change in phonology after HVC microlesions (i.e., large $\mathrm{K}-\mathrm{L}$ distance between Pre1 and Post1). Post2 K-L distance values for $\mathrm{HVCml}$ + LMANcs birds remained large, indicating sustained dissimilarity in phonology from Pre1. During Post2-8, phonology recovered gradually. This result replicates our previous report on the effects of HVC microlesions (Thompson and Johnson, 2007). However, Figure $9 B$ shows that LMAN ablation after HVC microlesions produced a precipitous decline in $\mathrm{K}-\mathrm{L}$ distance values, indicating rapid restoration of phonology at Post 2 that stabilized by Post3.

For HVCcs + LMAN Ablation birds, the removal of LMAN had only a modest impact on phonology for all spectral features. Here, there were only slight increases in the dissimilarity on Post 1 and Post2. Comparatively, these increases in dissimilarity were not nearly of the same magnitude as the dissimilarity that followed HVC microlesions (Fig. 9A-C, compare Post1, Post2). Statistical analysis of mean $\mathrm{K}-\mathrm{L}$ distance values revealed that these postoperative changes in phonology were not significant $\left(F_{(3,19)}=1.79 ; p=0.19\right)$ (Fig. $\left.7 C\right)$.

Both HVCml + LMAN Ablation and HVCml + LMANcs groups exhibited vocal recovery. Therefore, their behavior was characterized by a recovery curve derived from the K-L distance analysis of phonology. The recovery curve for each of these two groups was fit by an exponential decay function for days Post1-8

\section{A $\mathrm{HVCml}+$ LMANcs}

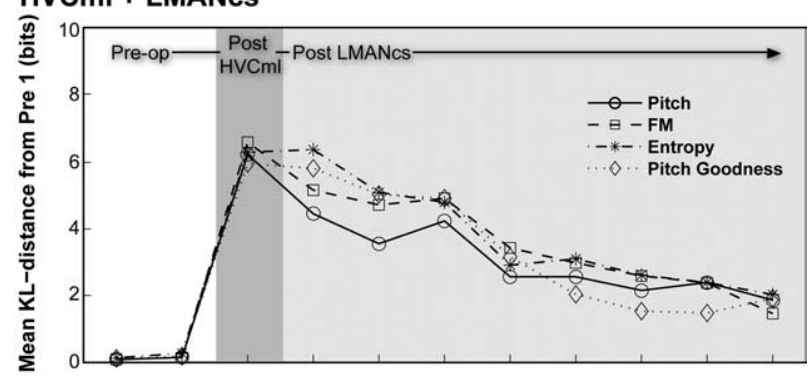

\section{B $\mathrm{HVCml}+$ LMAN Ablation}

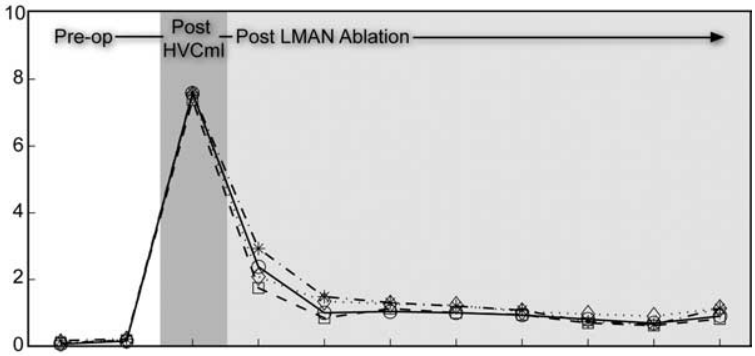

\section{HVCcs + LMAN Ablation}

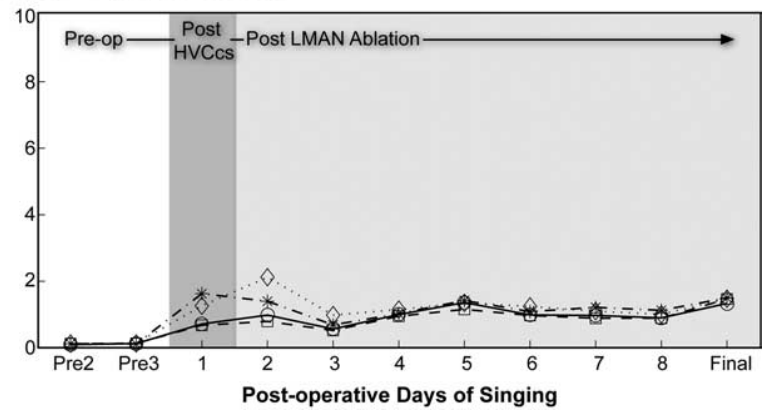

Figure 9. Mean K-L distance values from Pre1 describe changes in postoperative phonology for birds in the HVCml + LMANcs, HVCml + LMAN Ablation, and HVCcs + LMAN Ablation groups. Preoperative comparisons show little variation in phonology. $A$, HVC microlesions induced a marked increase in phonological dissimilarity that gradually declined during the course of the recovery period. $\boldsymbol{B}$, LMAN ablation after HVC microlesions caused a precipitous drop in dissimilarity from Post1 to Post2. Phonology stabilized by Post3. C, HVC control surgery and LMAN ablation caused a slight difference in phonology that stabilized by Post 3 and remained so to the end of recovery.

and Final, yielding a recovery time constant $\tau$ (expressed in units of days) (see Materials and Methods). A small $\tau$ indicates that recovery is rapid. For each bird, $\tau$ was calculated for each of the four spectral features (pitch, FM, entropy, and pitch goodness). Statistical analysis of mean $\tau$ values for each bird revealed that HVCml + LMAN Ablation birds (mean $\tau \pm$ SEM, $2.74 \pm 0.04 \mathrm{~d}$ ) recovered their preoperative song more rapidly than $\mathrm{HVCml}+$ LMANcs birds (mean $\tau \pm$ SEM, $6.48 \pm 0.86 \mathrm{~d} ; F_{(1,47)}=5.797 ; p<$ $0.05)$. Thus, LMAN ablation significantly accelerated the process of vocal recovery after HVC microlesions.

\section{Sudden vocal recovery after LMAN ablation: note sequencing} Figure $10 \mathrm{~A}$ shows sequence analysis for a representative bird from the HVCml + LMAN Ablation group (bird 610). Note transition probabilities at Pre1 were highest for transitions that included introductory notes and/or motif notes (compare with bird 610's frequency spectrograph and scatter plot in Fig. 2 A). At Post 1 , HVC microlesions produced an increase in the probability 
A HVCmI + LMAN Ablation (Bird 610)

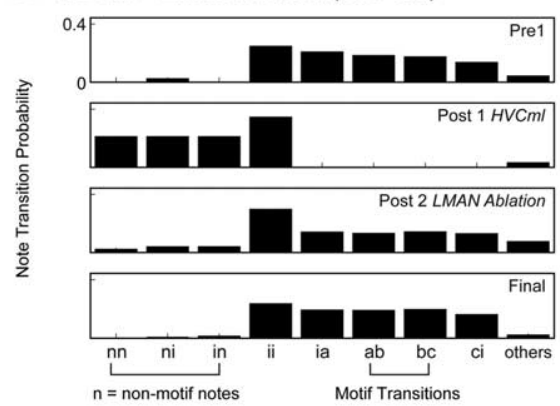

C $\mathrm{HVCml}+$ LMAN Ablation Birds

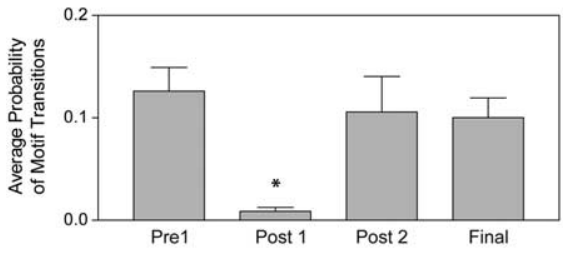

B $\mathrm{HVCml}+$ LMANcs (Bird 628)

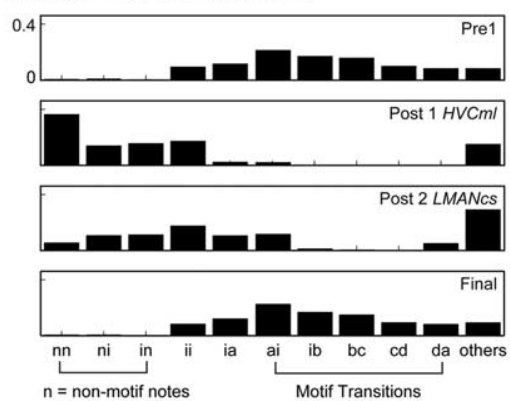

D $\mathrm{HVCml}+$ LMANcs Birds

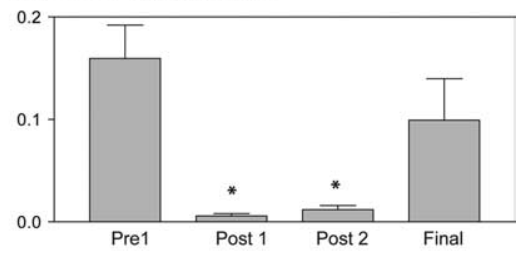

Figure 10. Note transition probabilities for $\mathrm{HVCml}+$ LMAN Ablation and $\mathrm{HVCml}+$ LMANcs groups. $\boldsymbol{A}$, Bird 610 shows note transition probabilities at Pre1 were primarily introductory note $(i)$ and motif note transitions (for description of "other" and "nonmotif note," see Materials and Methods). HVC microlesions (Post1 HVCml) abolished motif note transitions and induced an increase in transitions comprised of nonmotif notes. LMAN ablation (Post2 LMAN Ablation) restored preoperative note transition probabilities of motif notes. $\boldsymbol{B}$, Bird 628 shows that in the absence of LMAN ablation motif note transition probabilities were not immediately recovered. $C$, The average probability of motif note transitions from the $\mathrm{HVCml}+$ LMAN Ablation birds indicate that LMAN ablation induced a sudden restoration of note sequence after HVC microlesions. D, Although birds from the HVCml + LMANcs group recovered their note sequence by Final, few motif transitions were produced at Post1 and Post2. *Significant differences between days $(p<0.05)$. Error bars indicate SEM.

of transitions that contained nonmotif notes and a decrease in note transition probabilities associated with the motif. Subsequent to LMAN ablation, however, note transition probabilities that included nonmotif notes decreased and probabilities for note transitions within the motif increased; this increase was sustained to the end of the recovery period (see Post 2 and Final in Fig. 10A).

Conversely, when HVC microlesions were followed by control surgery (Figs. $2 \mathrm{~B}, 10 \mathrm{~B}$, bird 628), recovery of note transitions within the motif did not occur as rapidly. That is, at both Post1 and Post2, transition probabilities for notes within the motif were low and transitions composed of nonmotif notes were high. However, note transitions within the motif recovered by the last day of singing (Fig. $10 \mathrm{~B}$, Final).

To quantify the recovery of note sequence, we analyzed the average probability of motif transitions (i.e., note transitions that occur within the motif, which excludes introductory notes) at Pre1, Post1, Post2, and Final for each bird. A one-way ANOVA of the average probability of motif transitions for HVCml + LMAN Ablation birds resulted in a main effect $\left(F_{(3,23)}=4.783 ; p<0.05\right)$. Figure $10 C$ shows that there was a significant decrease in the probability of note transitions within the motif at Post1 when compared with Pre1, Post2, and Final (SNK test, all comparisons $p<0.05$ ). There was no difference between Pre1 and Post2 (or Final), indicating that note sequence recovered rapidly after LMAN ablation.

For HVCml + LMANcs birds, a one-way ANOVA revealed a main effect $\left(F_{(3,23)}=8.022 ; p<0.001\right)$. Figure $10 \mathrm{D}$ shows that there was a significant decrease in the probability of motif transitions during Post1 when compared with Pre1; however, this decrease persisted during Post2 (Pre1 vs Post1 and Pre1 vs Post2; SNK tests; both comparisons, $p<0.05)$. Post 1 was not different

from Post 2 , indicating that on both postoperative days of singing, the probability of motif transitions was equally low. However, Final and Pre1 were not different, indicating that motif sequence had recovered by the last day of singing (Fig. 10D).

\section{Discussion}

Our data reveal two main findings about vocal recovery after HVC microlesions. First, deafening after HVC microlesions prevented vocal recovery. Second, LMAN ablation after HVC microlesions facilitated rapid recovery of phonology (Fig. 9) and note sequence (Fig. 10). Thus, vocal recovery after $\mathrm{HVC}$ microlesions requires auditory feedback, but this recovery can be gradual (LMAN intact) or surprisingly abrupt (LMAN ablated). The latter finding provides additional support for our hypothesis that HVC microlesions lead to destabilized singing by weakening VMP input to RA, thereby favoring a vocalvariability signal carried by the AFP (Thompson and Johnson, 2007). However, our findings also address two key issues related to vocal plasticity: the role of auditory feedback and the role of the AFP.

\section{A novel role for auditory feedback: integration of VMP and AFP activity}

In both juveniles and adults, perturbation of auditory feedback leads to changes in the structure and sequence of notes. However, restoration of auditory function enables learning (juveniles) or recovery (adults) of song (Leonardo and Konishi, 1999; Funabiki and Konishi, 2003) (but cf. Zevin et al., 2004). Therefore, once juvenile birds establish a song template it appears that perturbed auditory feedback does not necessarily change or eliminate stored memories of song (which may exist at multiple locations), or prevent access to the song template in adulthood. Theoretical models developed to explain and predict the underlying mechanisms that enable vocal plasticity suggest that a computational mechanism compares the bird's singing to the song template and generates a Hebbian reinforcement signal when matches occur, constraining subsequent vocal production to the sounds present within the song template (Troyer and Doupe, 2000a,b; Troyer and Bottjer, 2001).

Although an auditory feedback mechanism for reinforcement of template-matched vocalizations seems necessary for juvenile learning, our findings reveal an additional role for auditory feedback that is focused solely on regulating the relative strength of VMP and AFP input to RA. That is, our present results show that auditory feedback is necessary for vocal recovery after HVC microlesions (Fig. 6) and that song recovers suddenly after LMAN ablation (Fig. 9). In previous work, we also found that, if LMAN is removed before HVC microlesions, birds will produce their preoperative song at their very first postoperative utterance (Thompson and Johnson, 2007). Together, these observations indicate that HVC microlesions do not impair the ability of the VMP to issue a coherent "motif" signal to RA, but this signaling is initially overridden by patterns of vocal variability generated by the AFP. Auditory feedback appears to enable vocal recovery by gradually strengthening VMP input, weakening AFP input, or perhaps both. 
Auditory feedback may therefore play two distinct but complementary roles during vocal development: (1) guiding the development of motor programming to achieve songs that match an encoded template and (2) gradually favoring the production of stereotyped song by reducing the influence of AFP activity in RA. Although these two processes would occur mostly in parallel during juvenile vocal development, vocal recovery after HVC microlesions may be driven primarily by a change in the relative strength of VMP and AFP input to RA, rather than a wholesale relearning of song (Fig. 11, model). Interestingly, this view provides an additional explanation for the findings of Brainard and Doupe (2000), who found that previous ablation of LMAN prevents deafening-induced destabilization of song. Although this report is often cited in support of template-based models, if auditory feedback regulates the relative strength of VMP and AFP input to RA (normally favoring VMP input), then deafening may contribute to song destabilization by allowing a gradual increase in ability of the AFP to drive a variable vocal output.

\section{Role of the AFP: instructive, motor, or both?}

Lesion studies in juveniles and adults demonstrate that vocal plasticity does not occur in the absence of LMAN (Bottjer et al., 1984; Scharff and Nottebohm, 1991; Morrison and Nottebohm, 1993; Williams and Mehta, 1999; Brainard and Doupe, 2000; Kao et al., 2005). Three models explain AFP function in vocal plasticity: an instructive model where auditory feedback reinforces the production of template-matched vocalizations (Troyer and Doupe, 2000a,b; Troyer and Bottjer, 2001), a purposive variation model in which motor variability facilitates trial-and-error learning of song (Kao et al., 2005; Ölveczky et al., 2005), and a permissive model in which AFP activity influences the release of plasticity factors (e.g., $\mathrm{BDNF}$ ) that enable morphological or synaptic changes within the VMP (Brainard, 2004). Conceptually, the three models are not mutually exclusive. However, we focus here on a comparison of instructive and purposive variability models; these two models make unambiguous and opposing predictions about the role of the AFP in vocal recovery after HVC microlesions.

Current knowledge limits the predictive value of the permissive model. We do not know whether an AFP-driven release of BDNF (or other plasticity factors) in RA occurs at the onset of vocal destabilization after HVC microlesions (cf. Kittelberger and Mooney, 2005), or whether such factors might also be released during the process of vocal recovery. Thus, it is not clear whether removal of such factors by LMAN ablation would prevent or facilitate vocal recovery.

\section{Instructive model}

During juvenile vocal development, the song control system shapes vocal output by guiding vocal approximations toward a desired goal (the song template) (Fig. 12A). The instructive model suggests that LMAN ablation impairs vocal development because the AFP con-

\section{B Adult Vocal Recovery}
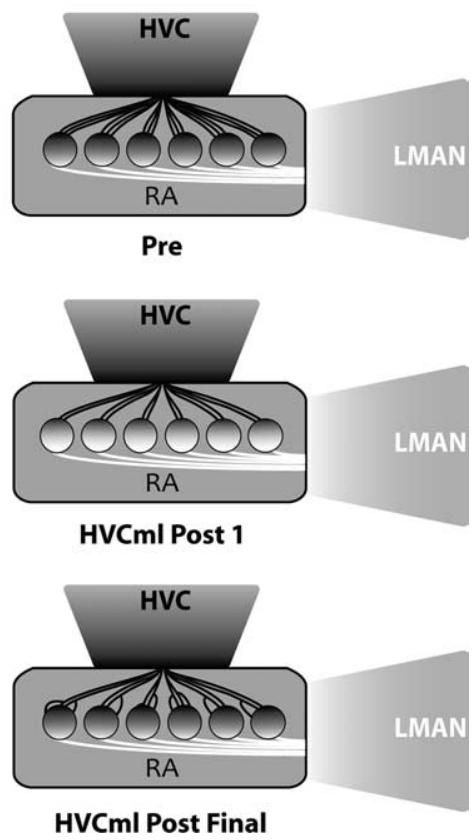

HVCml Post Final

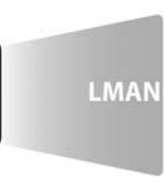

Juvenile Vocal Development
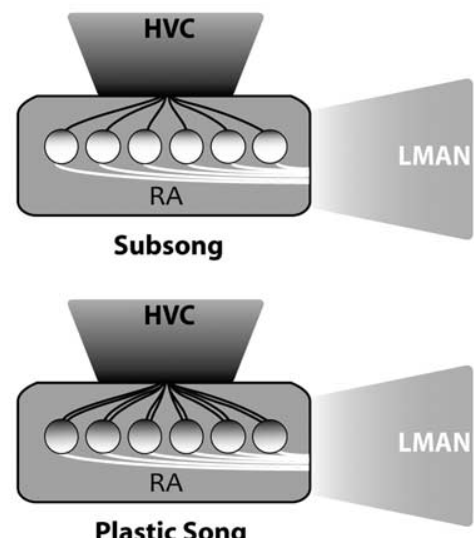

\section{(1)}

Figure 11. Simplified schematics show that similar changes in RA connectivity may underlie juvenile vocal development and a vocal recovery. $\boldsymbol{A}$, Stages of juvenile vocal development are paralleled by a gradual increase in HVC influence in RA through (Nordeen and Nordeen, 1988; Herrmann and Arnold, 1991). The number of LMAN neurons .

tains the computational mechanism that selectively biases VMP output toward sounds that match the template (Troyer and Doupe, 2000a,b; Troyer and Bottjer, 2001). Whereas LMAN ablation in adults produces only subtle effects on learned song (see below) (Kao et al., 2005), previous ablation of LMAN blocks deterioration of the vocal pattern caused by syringeal denervation or deafening (Williams and Mehta, 1999; Brainard and Doupe, 2000). Thus, the instructive model suggests that altered sensory-motor feedback in adult birds causes the AFP to bias VMP output in an aberrant direction.

In adult birds, neurons within the song control system respond selectively to playback of the bird's own song (BOS) under conditions of anesthesia or sleep (Doupe and Konishi, 1991; Dave and Margoliash, 2000; Hahnloser et al., 2006; Hahnloser and Fee, 2007). Selectivity for BOS responses in the AFP of juvenile birds emerges in parallel with behavioral development (Solis and Doupe, 1997, 1999). Thus, as the instructive model predicts, the AFP contains neurons that could use auditory feedback to recognize and reinforce the development of template-matched sounds. However, BOS responses within the AFP do not occur when birds are awake and singing, nor does motor-related AFP activity change in response to perturbation of auditory feedback (Hessler and Doupe, 1999; Leonardo, 2004; Kozhevnikov and Fee, 2007). Nevertheless, sleep-related (off-line) BOS responses may still guide feedback-based reinforcement of template-matched vocalizations (Dave and Margoliash, 2000; Hahnloser et al., 2006; Crandall et al., 2007; Hahnloser and Fee, 2007). Indeed, Deregnaucourt et al. (2005) demonstrate that during early vocal development 
A Juvenile Vocal Development (Bird 174)
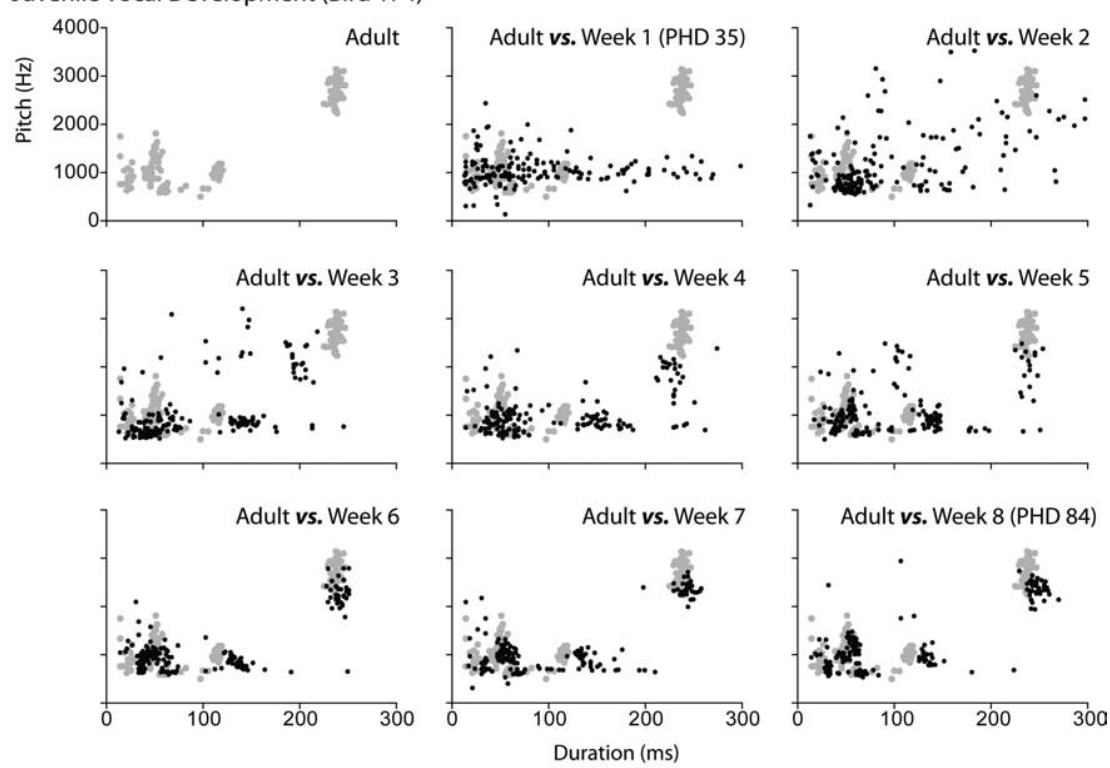

B Adult Vocal Recovery (Bird 557)
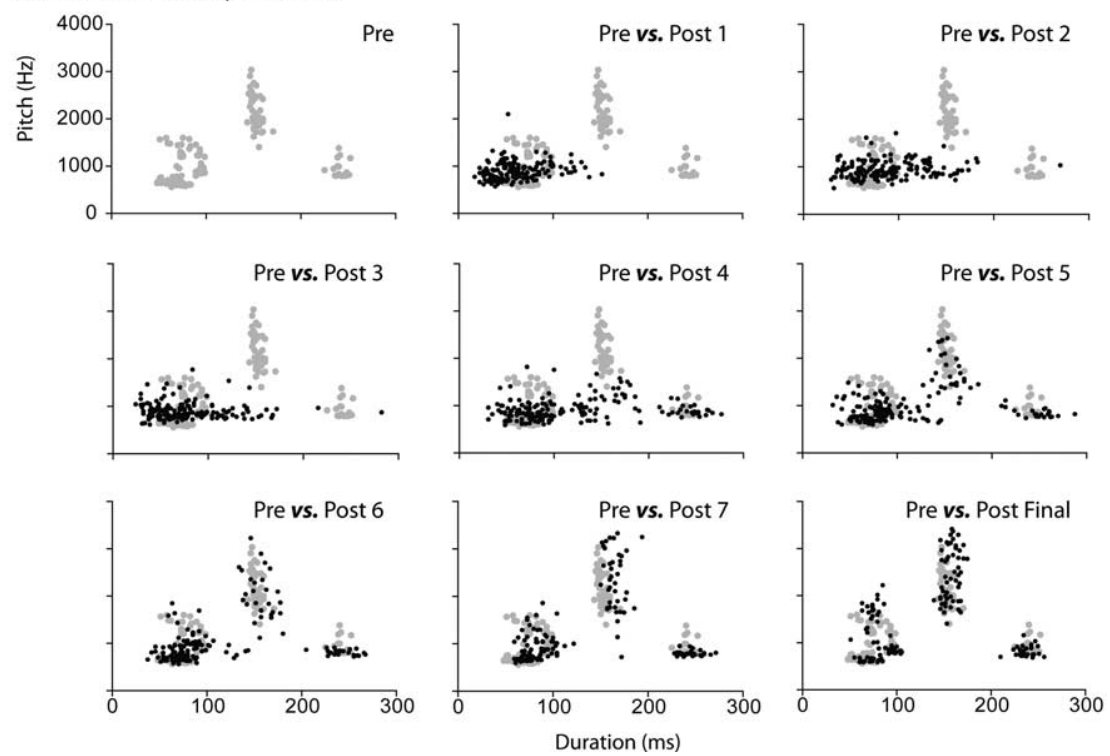

Figure 12. Duration versus pitch scatter plots compare juvenile vocal development and adult vocal recovery after HVC microlesions. $A$, Each scatter plot represents $1 \mathrm{~d}$ in each of the first 8 weeks of vocal development compared with a day of adult singing (20 randomly selected bouts from each day; the gray dots represent adult notes, and the black dots indicate developing notes). Notes produced during subsong (weeks 1,2) exhibit substantial variation in duration, but are initially limited in pitch. Plastic song (weeks 3-8) marks the gradual trend toward stereotypy, during which specific note types emerge. $\boldsymbol{B}$, Scatter plots compare consecutive days of postoperative singing after HVC microlesions with preoperative singing [20 randomly selected bouts from each day; the gray dots indicate preoperative notes, and the black dots represent postoperative notes; bird 557's data are from Thompson and Johnson (2007)]. Note phonology after HVC microlesions is similar to subsong; variable duration and limited pitch (Post1-3). Vocal recovery (Post4-Final) is similar to plastic song, although the emergence of stereotypy occurs more rapidly (time course of days instead of weeks).

an active process of sleep-related vocal plasticity may contribute to the emergence of structured sounds.

Vocal recovery after HVC microlesions requires auditory feedback and exhibits behavioral features that resemble juvenile vocal development (Fig. 12, compare $A, B$ ). Therefore, an instructive model of AFP function would predict that vocal recovery should be impaired by LMAN ablation. Instead, we found that LMAN ablation after HVC microlesions dramatically facilitated vocal recovery. These data effectively rule out the AFP as the neural locus of an instructive mechanism that guides adult vocal recovery after HVC microlesions.

\section{Purposive variation model}

The purposive variation model asserts that LMAN ablation impairs juvenile vocal development because the AFP generates variable patterns of vocal-motor activity necessary for trial-and-error learning of song (Kao et al., 2005; Ölveczky et al., 2005). In adult males, the AFP contributes motor variability to undirected song (Kao et al., 2005; Kao and Brainard, 2006), but AFP activity is suppressed during the production of highly stereotyped femaledirected courtship song (Jarvis et al., 1998; Hessler and Doupe, 1999; Kao and Brainard, 2006). Although the normal variation associated with undirected song is subtle, LMAN influences moment-to-moment variability in phonological structure of individual notes (Kao et al., 2005).

We hypothesized that HVC microlesions destabilize song by weakening VMP input to $\mathrm{RA}$, thereby increasing the influence of the AFP on vocal output (Thompson and Johnson, 2007). Thus, interpreted in light of the purposive variation model of AFP function, LMAN ablation should restore a pattern of RA connectivity that favors VMP input and facilitates vocal recovery. Our results confirm this interpretation (Figs. 9, 10), providing strong support for the purposive variation model of AFP function (Kao et al., 2005; Ölveczky et al., 2005). Thus, during periods of adult vocal change, the AFP appears to generate vocal-motor variation, rather than an instructive signal.

Although the contribution of the AFP to vocal variability in adult zebra finches is modest and of unknown behavioral significance, many songbird species show seasonal relearning and/or modification of song in adulthood (Brenowitz and Beecher, 2005), and in these species the AFP may play an adult role that is similar to that seen in juvenile zebra finches. More generally, our results also support an emerging view that corticobasal ganglia circuits provide the motor variability necessary to acquire new behavioral sequences through a process of trial-anderror learning (Graybiel, 2005).

\section{References}

Bottjer SW, Miesner EA, Arnold AP (1984) Forebrain lesions disrupt development but not maintenance of song in passerine birds. Science 224:901-903.

Brainard MS (2004) Contributions of the anterior forebrain pathway to vocal plasticity. Ann NY Acad Sci 1016:377-394.

Brainard MS, Doupe AJ (2000) Interruption of a basal ganglia-forebrain circuit prevents plasticity of learned vocalizations. Nature 404:762-766.

Brainard MS, Doupe AJ (2001) Postlearning consolidation of birdsong: stabilization effects of age and anterior forebrain lesions. J Neurosci 21:2501-2517.

Brenowitz EA, Beecher MD (2005) Song learning in birds: diversity and plasticity, opportunities and challenges. Trends Neurosci 28:127-132.

Canady RA, Burd GD, DeVoogd TJ, Nottebohm F (1988) Effect of testosterone 
on input received by an identified neuron type of the canary song system: a Golgi/electron microscopy/degeneration study. J Neurosci 8:3770-3784.

Coleman MJ, Mooney R (2004) Synaptic transformations underlying highly selective auditory representations of learned birdsong. J Neurosci 24:7251-7265.

Crandall SR, Adam M, Kinnischtzke AK, Nick TA (2007) HVC neural sleep activity increases with development and parallels nightly changes in song behavior. J Neurophysiol 98:232-240.

Cynx J, von Rad U (2001) Immediate and transitory effects of delayed auditory feedback on bird song production. Anim Behav 62:305-312.

Dave AS, Margoliash D (2000) Song replay during sleep and computational rules for sensorimotor vocal learning. Science 290:812-816.

Deregnaucourt S, Mitra PP, Feher O, Pytte C, Tchernichovski O (2005) How sleep affects the developmental learning of bird song. Nature 433:710-716.

Doupe AJ, Konishi M (1991) Song-selective auditory circuits in the vocal control system of zebra finches. Proc Natl Acad Sci USA 88:11339-11343.

Dutar P, Vu HM, Perkel DJ (1998) Multiple cell types distinguished by physiological, pharmacological, and anatomic properties in nucleus HVc of the adult zebra finch. J Neurophysiol 80:1828-1838.

Foster EF, Bottjer SW (1998) Axonal connections of the high vocal center and surrounding cortical regions in juvenile and adult male zebra finches. J Comp Neurol 397:118-138.

Funabiki Y, Konishi M (2003) Long memory in song learning by zebra finches. J Neurosci 23:6928-6935.

Graybiel AM (2005) The basal ganglia: learning new tricks and loving it. Curr Opin Neurobiol 15:638-644.

Hahnloser RH, Fee MS (2007) Sleep-related spike bursts in HVC are driven by the nucleus interface of the nidopallium. J Neurophysiol 97:423-435.

Hahnloser RH, Kozhevnikov AA, Fee MS (2002) An ultra-sparse code underlies the generation of neural sequences in a songbird. Nature 419:65-70.

Hahnloser RH, Kozhevnikov AA, Fee MS (2006) Sleep-related neural activity in a premotor and a basal-ganglia pathway of the songbird. J Neurophysiol 96:794-812.

Herrmann K, Arnold AP (1991) The development of afferent projections to the robust archistriatal nucleus in male zebra finches: a quantitative electron microscopic study. J Neurosci 11:2063-2074.

Hessler NA, Doupe AJ (1999) Singing-related neural activity in a dorsal forebrain-basal ganglia circuit of adult zebra finches. J Neurosci 19:10461-10481.

Jarvis ED, Scharff C, Grossman MR, Ramos JA, Nottebohm F (1998) For whom the bird sings: context-dependent gene expression. Neuron 21:775-788.

Johnson F, Sablan MM, Bottjer SW (1995) Topographic organization of a forebrain pathway involved with vocal learning in zebra finches. J Comp Neurol 358:260-278.

Kao MH, Brainard MS (2006) Lesions of an avian basal ganglia circuit prevent context-dependent changes to song variability. J Neurophysiol 96:1441-1455.

Kao MH, Doupe AJ, Brainard MS (2005) Contributions of an avian basal ganglia-forebrain circuit to real-time modulation of song. Nature 433:638-643.

Kittelberger JM, Mooney R (2005) Acute injections of brain-derived neurotrophic factor in a vocal premotor nucleus reversibly disrupt adult birdsong stability and trigger syllable deletion. J Neurobiol 62:406-424.

Kozhevnikov AA, Fee MS (2007) Singing-related activity of identified HVC neurons in the zebra finch. J Neurophysiol 97:4271-4283.

Kullback S, Leibler RA (1951) On information and sufficiency. Ann Math Statist 22:49-86.

Leonardo A (2004) Experimental test of the birdsong error-correction model. Proc Natl Acad Sci USA 101:16935-16940.

Leonardo A, Konishi M (1999) Decrystallization of adult birdsong by perturbation of auditory feedback. Nature 399:466-470.

Lombardino AJ, Nottebohm F (2000) Age at deafening affects the stability of learned song in adult male zebra finches. J Neurosci 20:5054-5064.

Mooney R (2000) Different subthreshold mechanisms underlie song selectivity in identified $\mathrm{HVc}$ neurons of the zebra finch. J Neurosci 20:5420-5436.

Mooney R, Konishi M (1991) Two distinct inputs to an avian song nucleus activate different glutamate receptor subtypes on individual neurons. Proc Natl Acad Sci USA 88:4075-4079.

Mooney R, Prather J (2005) The HVC microcircuit: the synaptic basis for interactions between song motor and vocal plasticity pathways. J Neurosci 25:1952-1964.

Morrison RG, Nottebohm F (1993) Role of a telencephalic nucleus in the delayed song learning of socially isolated zebra finches. J Neurobiol 24:1045-1064.

Nordeen EJ, Grace A, Burek MJ, Nordeen KW (1992) Sex-dependent loss of projection neurons involved in avian song learning. J Neruobiol 23:671-679.

Nordeen KW, Nordeen EJ (1988) Projection neurons within a vocal motor pathway are born during song learning in zebra finches. Nature 334:149-151.

Nordeen KW, Nordeen EJ (1992) Auditory feedback is necessary for the maintenance of stereotyped song in adult zebra finches. Behav Neural Biol 57:58-66.

Ölveczky BP, Andalman AS, Fee MS (2005) Vocal experimentation in the juvenile songbird requires a basal ganglia circuit. PLoS Biol 3:e153.

Riede T, Suthers RA, Fletcher NH, Blevins WE (2006) Songbirds tune their vocal tract to the fundamental frequency of their song. Proc Natl Acad Sci USA 103:5543-5548.

Scharff C, Nottebohm F (1991) A comparative study of the behavioral deficits following lesions of various parts of the zebra finch song system: implications for vocal learning. J Neurosci 11:2896-2913.

Scharff C, Kirn JR, Grossman M, Macklis JD, Nottebohm F (2000) Targeted neuronal death affects neuronal replacement and vocal behavior in adult songbirds. Neuron 25:481-492.

Simpson HB, Vicario DS (1990) Brain pathways for learned and unlearned vocalizations differ in zebra finches. J Neurosci 10:1541-1556.

Solis MM, Doupe AJ (1997) Anterior forebrain neurons develop selectivity by an intermediate stage of birdsong learning. J Neurosci 17:6447-6462.

Solis MM, Doupe AJ (1999) Contributions of tutor and bird's own song experience to neural selectivity in the songbird anterior forebrain. J Neurosci 19:4559-4584.

Sossinka R, Bohner J (1980) Song types in the zebra finch (Poephila guttata castanotis). Z Tierpsychol 53:123-132.

Stark LL, Perkel DJ (1999) Two stage, input-specific synaptic maturation in a nucleus essential for vocal production in the zebra finch. J Neurosci 19:9107-9116.

Tchernichovski O, Nottebohm F, Ho CE, Pesaran B, Mitra PP (2000) A procedure for an automated measurement of song similarity. Anim Behav 59:1167-1176.

Thompson JA, Johnson F (2007) HVC microlesions do not destabilize the vocal patterns of adult male zebra finches with prior ablation of LMAN. Dev Neurobiol 67:205-218.

Troyer TW, Bottjer SW (2001) Birdsong: models and mechanisms. Curr Opin Neurobiol 11:721-726.

Troyer TW, Doupe AJ (2000a) An associational model of birdsong sensorimotor learning I. Efference copy and the learning of song syllables. J Neurophysiol 84:1204-1223.

Troyer TW, Doupe AJ (2000b) An associational model of birdsong sensorimotor learning II. Temporal hierarchies and the learning of song sequence. J Neurophysiol 84:1224-1239.

Vates GE, Broome BM, Mello CV, Nottebohm F (1996) Auditory pathways of caudal telencephalon and their relation to the song system of adult male zebra finches. J Comp Neurol 366:613-642.

Wang N, Aviram R, Kirn JR (1999) Deafening alters neuron turnover within the telencephalic motor pathway for song control in adult zebra finches. J Neurosci 19:10554-10561.

Watanabe A, Li R, Kimura T, Sakaguchi H (2006) Lesions of an avian forebrain nucleus prevent changes in protein kinase $\mathrm{C}$ levels associated with deafening-induced vocal plasticity in adult songbirds. Eur J Neurosci 23:2447-2457.

Wild JM, Williams MN, Howie GJ, Mooney R (2005) Calcium-binding proteins define interneurons in HVC of the zebra finch (Taeniopygia guttata). J Comp Neurol 483:76-90.

Williams H, Mehta N (1999) Changes in adult zebra finch song require a forebrain nucleus that is not necessary for song production. J Neurobiol 39:14-28.

Yu AC, Margoliash D (1996) Temporal hierarchical control of singing in birds. Science 273:1871-1875.

Zevin JD, Seidenberg MS, Bottjer SW (2004) Limits on reacquisition of song in adult zebra finches exposed to white noise. J Neurosci 24:5849-5862. 